\title{
MAGNETIC PHOTON SPLITTING: THE S-MATRIX FORMULATION IN THE LANDAU REPRESENTATION
}

\author{
Matthew G. Baring* \\ Laboratory for High Energy Astrophysics, Code 661, \\ NASA Goddard Space Flight Center, Greenbelt, MD 20771, USA
}

(Accepted for pubmication in Physical Review D)

\begin{abstract}
Calculations of reaction rates for the third-order QED process of photon splitting $\gamma \rightarrow \gamma \gamma$ in strong magnetic fields traditionally have employed either the effective Lagrangian method or variants of Schwinger's proper-time technique. Recently, Mentzel, Berg and Wunner [⿰] [- presented an alternative derivation via an S-matrix formulation in the Landau representation. Advantages of such a formulation include the ability to compute rates near pair resonances above pair threshold. This paper presents new developments of the Landau representation formalism as applied to photon splitting, providing significant advances beyond the work of [1] by summing over the spin quantum numbers of the electron propagators, and analytically integrating over the component of momentum of the intermediate states that is parallel to field. The ensuing tractable expressions for the scattering amplitudes are satisfyingly compact, and of an appearance familiar to S-matrix theory applications. Such developments can facilitate numerical computations of splitting considerably both below and above pair threshold. Specializations to two regimes of interest are obtained, namely the limit of highly supercritical fields and the domain where photon energies are far inferior to that for the threshold of single-photon pair creation. In particular, for the first time the low-frequency amplitudes are simply expressed in terms of the Gamma function, its integral and its derivatives. In addition, the equivalence of the asymptotic forms in these two domains to extant results from effective Lagrangian/proper-time formulations is demonstrated.
\end{abstract}

12.20.Ds, 95.30.Cq, 97.60.Gb, 97.60.Jd, 98.70.Rz

\section{INTRODUCTION}

The third-order quantum electrodynamical process of photon splitting $\gamma \rightarrow \gamma \gamma$ in a strong magnetic field, currently popular in several astrophysical models of different neutron star sources, was first studied over three decades ago. Due to analytic complexities encountered when investigating this interaction, it was not until the beginning of the 1970s that a body of correct and uncontroversial results emerged. These early splitting calculations used either effective Lagrangian [2, 4] or variations of Schwinger's proper-time techniques [5], the expediency of which yielded compact analytic forms for the rates $R$ when specializing to low energy $\left(R \propto \omega^{5}\right)$ or low field $\left(R \propto B^{6}\right)$ cases. After a hiatus of nearly two decades, photon splitting became of interest again in the literature [8 11 following the publication of an S-matrix calculation in the Landau representation of its rates by Mentzel, Berg and Wunner [1], specifically because of their contention that the earlier works cited above had seriously underestimated the strength of this process. The rates computed in [1] were later retracted in [12], with a sign error in their numerical coding having been discovered and corrected. Mentzel et al.'s analytic derivation was the first comprehensive presentation of the application of a Landau representation technique specifically to magnetic photon splitting, though the QED formalism presented by Melrose and Parle 13 14 virtually provided an equivalent enunciation of such S-matrix forms for splitting amplitudes. More recently, Weise, Baring \& Melrose [1] confirmed the analytic derivation of [1]. The Landau representation calculations and most of the earlier effective Lagrangian and proper-time presentations were generally applicable to non-dispersive regimes below the pair creation threshold ( $\left.\hbar \omega=2 m c^{2}\right)$, where the momentum vectors of the initial and final photons are collinear, and arbitrary field strengths.

Below pair threshold, the effective Lagrangian approach of [2, 4] and the proper-time calculations in [5 8] appear much more amenable for the purposes of numerical evaluation than the S-matrix formulation in the Landau representation. This arises because effective Lagrangian and proper-time (collectively referred to by the label ELP here) methods produce results that involve triple integrals over relatively simple (hyperbolic and exponential) functions, while the S-matrix amplitudes integrate over the parallel momentum $p_{z}$ and include a triple summation over the

*Universities Space Research Association. Email: baring@lheavx.gsfc.nasa.gov 
Landau level quantum numbers of the intermediate pair states. Both techniques start from different but equivalent $115]$ forms of the electron propagator, and hence S-matrix computations [1.11] should yield identical results to propertime numerics [2 8]. For the specific case of magnetic pair creation $\gamma \rightarrow e^{+} e^{-}$, such an equivalence of the S-matrix and proper-time methods has been demonstrated [16.17], but only via continuous asymptotic approximations that smoothly average out the exact "sawtooth" resonance structure. Yet the S-matrix Landau representation approach explicitly retains the resonances in the scattering amplitudes above pair threshold, whereas the ELP methods eliminate such information early during developments. Photon splitting becomes effectively first-order in $\alpha_{\mathrm{f}}$ at any one of a multitude of pair resonances, generated when the intermediate states become "on-shell." Hence it is quite possible that splitting can compete effectively with pair creation as a photon absorption mechanism above pair threshold. Ascertaining whether this is true is an interesting physics question. Moreover, if splitting is approximately as probable as pair creation above threshold, then it manifestly changes the character of vacuum dispersion, so that quadratic (and by inference perhaps higher order) contributions to the vacuum polarization tensor become significant relative to the standard linear ones used in the derivation [4] of kinematic selection rules for splitting. Hence, the generation of exact and compact expressions for the rates for $\gamma \rightarrow \gamma \gamma$ valid both below and above pair creation threshold is clearly a worthwhile enterprise from a physics perspective.

Developed expressions for the rates for photon splitting are also important for astrophysical applications of this process, particularly to effect efficient and accurate computations of such rates. These applications have so far focused on neutron star magnetospheres, primarily on models of soft gamma repeaters (SGRs) and strongly-magnetized pulsars, both being extremely topical in the astrophysics community at present. The potential importance of splitting in neutron star environments was suggested by [4, 18, 19]. Possible formation of splitting cascades has been explored in models of SGR transient outbursts as a means of softening the spectrum efficiently with no production of pairs [20 23. If both polarizations can split, or if polarization switching is active during SGR outbursts, then the properties of the splitting cross-section guarantee emergent spectra in the observed range (20-150 keV) and of the observed shape for all fields in excess of around $10^{14}$ Gauss [20 22], provided that the emission region is not concentrated near the polar cap. The spectral properties of SGRs in quiescent emission appear to be distinct from those during outburst. Pulsations and temporal increases of their periods (i.e. spin-down) have now been observed 24 26] for two of the four confirmed SGRs (SGR 1806-20 and SGR 1900+14), leading to inferences of fields in the vicinity of $10^{15}$ Gauss. The connection between these pulsars of extremely high magnetization, so-called magnetars, and conventional radio/Xray/gamma-ray pulsars is not well-understood. Baring \& Harding [27] postulated that radio quiescence, a property of the SGRs, may be common in magnetars due to the efficient action of photon splitting and other effects in suppressing the creation of pairs. Photon splitting also has spectral implications for such pulsars with more modest fields: [28] demonstrated that the unusual absence of $>30 \mathrm{MeV}$ emission in the gamma-ray pulsar PSR 1509-58 (whose spindown field is $\sim 3 \times 10^{13}$ Gauss) can naturally be explained by the operation of $\gamma \rightarrow \gamma \gamma$ in the intense magnetic and gravitational fields near its surface.

Several desirable goals are immediately identifiable on the basis of this historical path for the study of the physics of photon splitting, and the needs of the astrophysics community. It would be satisfying (i) to obtain analytic expressions for rates that are valid above pair creation threshold using the Landau representation methodology, (ii) to know whether the analytic formalism of Mentzel et al. [1] can be developed and simplified, and (iii) to demonstrate a formal equivalence between this S-matrix Landau representation approach and extant results from proper-time/effective Lagrangian techniques. This paper addresses these issues, using the verified analytic formalism of Mentzel et al. as the starting point for mathematical developments. The analysis here considers all the polarization modes that are permitted by the CP invariance symmetry $(\perp \rightarrow\|\|, \perp \rightarrow \perp \perp$ and $\|\rightarrow \perp\|)$, and applies for collinear momenta of the incoming and outgoing photons, i.e. when the effects of vacuum dispersion are neglected. A significant development provided in this paper is the dramatic simplification incurred by algebraically performing the summation over the spin states that are incorporated in the electron propagators. The resulting expressions in Section II A (first stated in [11]) are relatively compact, and of an appearance familiar to Landau representation/S-matrix theory applications to magnetized environments (i.e. including associated Laguerre functions). Furthermore, here the integrations over the momentum parallel to the field are performed analytically for the first time in Section IIB, rendering the splitting rates in most amenable forms (see Eqs. [12] and [15]) that are optimal for numerical applications: the analytic forms presented consist of just triple summations over Landau level quantum numbers of the intermediate states. These general results are valid both below and above pair threshold at non-resonant photon energies, and provide substantial advances over the work of [1]; they are much more suitable for numerical evaluation since many cancellations have been eliminated algebraically.

Two specializations are discussed in Section III, primarily to (partially) demonstrate equivalence of the Landau representation formalism presented here with extant proper-time/effective Lagrangian limiting forms for splitting rates, and simultaneously to serve as a check on the mathematical manipulations of this paper. Results are presented for all three polarization modes permitted by $\mathrm{CP}$ invariance in the limit of zero dispersion. The first asymptotic regime is (see Section III A) for highly supercritical fields, $B \gg B_{\mathrm{c}}=m^{2} c^{3} / e \hbar$, where in the case of $\perp \rightarrow\|\|$, the limit 
was found to concur with a recent analytic result that was obtained by Baier et al. [8], while new results were obtained for the other two modes. In the second specialization, in Section III B, asymptotic results for energies $\omega \ll m c^{2}$ well below pair creation threshold were obtained, reproducing the cubic energy dependence of the amplitudes obtained by other QED techniques. Moreover, new and compact expressions for the scattering amplitudes in this low energy limit are derived in terms of the logarithm of the Gamma function, its integral and their derivatives. These simplified forms in Eqs. (41) and (42) are also produced from extant integral forms for splitting matrix elements derived first in [2. 4 , thereby facilitating the first analytic demonstration of the equivalence of splitting rates obtained by the S-matrix formulation in the Landau representation and those derived using Schwinger-type techniques.

\section{THE GENERAL S-MATRIX FORMALISM}

The rates for photon splitting within an S-matrix formulation can be developed using a variety of conventions; here the Landau representation used by Mentzel, Berg and Wunner [1] is adopted, and formal developments lead to an independent confirmation of their analytic derivation. Specifically, for a field $\mathbf{B}=(0,0, B)$, this approach uses a representation of the electron/positron wavefunctions as eigenstates of the magnetic moment (or spin) operator $\mu_{z}$ (with $\boldsymbol{\mu}=m \boldsymbol{\sigma}+\gamma_{5} \beta \boldsymbol{\sigma} \times[\mathbf{p}+e \mathbf{A}(\mathbf{x})]$ ) in Cartesian coordinates within the confines of the Landau gauge $\mathbf{A}(\mathbf{x})=$ $(0, B x, 0)$. Such states turn out to be very convenient because they generate useful symmetry properties; they were identified by Sokolov and Ternov [29], who dubbed them states of "transverse polarization." Let $e, e^{\prime}$ and $e^{\prime \prime}$ denote the polarizations of the initial and final (primed) photons $\left(e, e^{\prime}, e^{\prime \prime}=\perp, \|\right)$, and $k_{\mu}=(\omega, \mathbf{k}), k_{\mu}^{\prime}=\left(\omega^{\prime}, \mathbf{k}^{\prime}\right)$ and $k_{\mu}^{\prime \prime}=\left(\omega^{\prime \prime}, \mathbf{k}^{\prime \prime}\right)$ denote the absorbed and produced photon four momenta. Then the total rate for splitting via the polarization mode $e \rightarrow e^{\prime} e^{\prime \prime}$ can be written, using Eqs. (27)-(29) of [1], in terms of the S-matrix element $S_{f i}^{(3)}$, which is the sum of six terms $S_{f i, j}^{(3)}$ corresponding to the six viable time-ordering possibilities:

$$
R_{e \rightarrow e^{\prime} e^{\prime \prime}}=\frac{1}{2}\left(\frac{V}{8 \pi^{3}}\right)^{2} \frac{m c^{2}}{\hbar} \int d^{3} k^{\prime} d^{3} k^{\prime \prime} \frac{1}{T}\left|\sum_{j=1,6} S_{f i, j}^{(3)}\right|^{2},
$$

where $V$ and $T$ denote the volume and time associated with the interaction calculation and the factor of $1 / 2$ out the front avoids double counting of the final states. The priming convention adopted throughout the paper is one and two primes for the produced photons and no prime for the initial photon. Since the S-matrix element contains a delta function $\delta^{4}\left(k_{\mu}-k_{\mu}^{\prime}-k_{\mu}^{\prime \prime}\right)$ prescribing four-momentum conservation for splitting, it is squared in the usual way using $\left|\delta^{4}\left(k_{\mu}-k_{\mu}^{\prime}-k_{\mu}^{\prime \prime}\right)\right|^{2} \rightarrow\left[V T /(2 \pi)^{4}\right] \delta^{4}\left(k_{\mu}-k_{\mu}^{\prime}-k_{\mu}^{\prime \prime}\right)$. Note that the S-matrix element should possess a cubic dependence on photon energies when well below pair creation threshold, due to parity symmetry, photon gauge invariance, and the antisymmetric nature of the electromagnetic field tensor; details are discussed in $\llbracket$.

Before writing down expressions for the S-matrix element terms, it is appropriate to identify the dimensionless convention that shall be adopted throughout this paper. Since the electron rest mass $m$ is the only mass that enters into this QED problem, we opt to scale all energies by $m c^{2}$ and momenta by $m c$ unless otherwise specified. This includes a scaling of $m c^{2} / \hbar$ for photon frequencies $\omega$. In the spirit of this convention, we choose to use the symbol $\varepsilon$ to represent dimensionless electron energies and reserve $E\left(=\varepsilon m c^{2}\right)$ to denote "dimensional" energies as in [i]. In addition, the magnetic field will be expressed in terms of the quantum critical field $B_{\mathrm{c}}=m^{2} c^{3} / e \hbar$ hereafter, so that $B=1$ denotes a field of $4.413 \times 10^{13}$ Gauss.

The convention for polarizations is identical to that assumed in [1], who opted for real polarization vectors with zero time components. The polarization states $\perp$ and $\|$ are defined according to whether the photon's electric vector lies either perpendicular or parallel (respectively) to the plane containing the photon's momentum $\mathbf{k}$ and the (uniform) magnetic field $\mathbf{B}$ vectors, the convention of [1, 13, 6, 16, 10, 30]. In the limit of zero dispersion, three polarization modes are permitted by charge/parity (CP) invariance in QED, namely $\perp \rightarrow\|\|, \perp \rightarrow \perp \perp$ and $\|\rightarrow \perp\|$. However, Adler [4] showed (see also [31]) that for weak vacuum dispersion (roughly delineated by $B \lesssim 1$ ), where the refractive indices for the polarization states are very close to unity, energy and momentum could simultaneously be conserved only for the splitting mode $\perp \rightarrow\|\|$. This kinematic selection rule applies to gamma-ray pulsar magnetospheres where plasma dispersion is negligible. In magnetar models of soft gamma repeaters, where supercritical fields are employed, strong vacuum dispersion arises. In such a regime, it is not clear whether Adler's selection rules still endure, since his linear dispersion analysis omits higher order (quadratic) contributions [13,14] to the vacuum polarization tensor (e.g. those that couple to photon absorption via splitting) that may become significant in supercritical fields. Furthermore, plasma dispersion effects, which can nullify the vacuum selection rules, may be quite pertinent [32] to soft gamma repeater magnetospheres, rendering them distinctly different from those of conventional pulsars. Therefore, in the interests of generality, consideration of all three CP-permitted splitting modes is adopted throughout this paper. 
The derivation of the S-matrix element proceeds along lines identical to those in Mentzel, Berg \& Wunner [1], with the result being an exact reproduction of their analytic formalism, as reported in Weise, Baring \& Melrose [11]; for details, one is referred to 1 . Of the six $S_{f i, j}^{(3)}$ contributions to Eq. (11), it is sufficient to explicitly present just one:

$$
\begin{aligned}
S_{f i, 1}^{(3)} & =-i \frac{\pi^{2}}{16} \frac{\left(4 \pi \alpha_{\mathrm{f}}\right)^{3 / 2} B}{\sqrt{\omega \omega^{\prime} \omega^{\prime \prime}}} \frac{1}{(2 V)^{3 / 2}} \delta^{(4)}\left(k_{\mu}-k_{\mu}^{\prime}-k_{\mu}^{\prime \prime}\right) \sum_{n n^{\prime} n^{\prime \prime}} \sum_{\sigma \sigma^{\prime} \sigma^{\prime \prime}} \frac{1}{\varepsilon_{0} \varepsilon_{0}^{\prime} \varepsilon_{0}^{\prime \prime}} \int d p_{z} \\
& \times\left.\frac{\mathcal{D}_{n^{\prime} n}^{++}\left(\mathbf{k}^{\prime \prime}\right) \mathcal{D}_{n n^{\prime \prime}}^{+-}\left(\mathbf{k}^{\prime}\right) \mathcal{D}_{n^{\prime \prime} n^{\prime}}^{-+}(\mathbf{k})-\mathcal{D}_{n n^{\prime}}^{--}\left(\mathbf{k}^{\prime \prime}\right) \mathcal{D}_{n^{\prime \prime} n}^{+-}\left(\mathbf{k}^{\prime}\right) \mathcal{D}_{n^{\prime} n^{\prime \prime}}^{-+}(\mathbf{k})}{\varepsilon \varepsilon^{\prime} \varepsilon^{\prime \prime}\left(\varepsilon+\varepsilon^{\prime \prime}+\omega^{\prime}-i \epsilon\right)\left(\varepsilon^{\prime}+\varepsilon^{\prime \prime}+\omega-i \epsilon^{\prime}\right)}\right|_{p_{z}^{\prime}=p_{z}-k_{z}^{\prime \prime}, p_{z}^{\prime \prime}=-p_{z}-k_{z}^{\prime}},
\end{aligned}
$$

where $\alpha_{\mathrm{f}}=e^{2} /(\hbar c)$ is the fine structure constant, and the energies $\varepsilon$ and $\varepsilon_{0}$ are defined in Eq. (疋) below, with similar definitions for the primed energies involving primed quantum numbers and momenta of the virtual electrons. Using the $J$ notation in Eq. (6) below,

$$
\begin{aligned}
\mathcal{D}_{n^{\prime} n}^{++}\left(\mathbf{k}^{\prime \prime}\right)= & J\left(-k_{x}^{\prime \prime}\left|n^{\prime}-1,-k_{y}^{\prime \prime}\right| n, 0\right)\left[\kappa_{1}^{\prime *} \kappa_{4}+\kappa_{3}^{\prime *} \kappa_{2}\right] e_{-}^{\prime \prime} \\
& +J\left(-k_{x}^{\prime \prime}\left|n^{\prime},-k_{y}^{\prime \prime}\right| n-1,0\right)\left[\kappa_{4}^{\prime *} \kappa_{1}+\kappa_{2}^{\prime *} \kappa_{3}\right] e_{+}^{\prime \prime} \\
& -\left\{J\left(-k_{x}^{\prime \prime}\left|n^{\prime},-k_{y}^{\prime \prime}\right| n, 0\right)\left[\kappa_{2}^{\prime *} \kappa_{4}+\kappa_{4}^{\prime *} \kappa_{2}\right]-J\left(-k_{x}^{\prime \prime}\left|n^{\prime}-1,-k_{y}^{\prime \prime}\right| n-1,0\right)\left[\kappa_{1}^{\prime *} \kappa_{3}+\kappa_{3}^{\prime *} \kappa_{1}\right]\right\} e_{z}^{\prime \prime}, \\
\mathcal{D}_{n n^{\prime \prime}}^{+-}\left(\mathbf{k}^{\prime}\right)= & J\left(-k_{x}^{\prime}|n-1,0| n^{\prime \prime}, k_{y}^{\prime}\right)\left[\kappa_{1}^{*} \kappa_{2}^{\prime \prime *}+\kappa_{3}^{*} \kappa_{4}^{\prime \prime *}\right] e_{-}^{\prime} \\
& +J\left(-k_{x}^{\prime}|n, 0| n^{\prime \prime}-1, k_{y}^{\prime}\right)\left[\kappa_{2}^{*} \kappa_{1}^{\prime \prime *}+\kappa_{4}^{*} \kappa_{3}^{\prime \prime *}\right] e_{+}^{\prime} \\
& -\left\{J\left(-k_{x}^{\prime}|n, 0| n^{\prime \prime}, k_{y}^{\prime}\right)\left[\kappa_{2}^{*} \kappa_{2}^{\prime \prime *}+\kappa_{4}^{*} \kappa_{4}^{\prime \prime *}\right]-J\left(-k_{x}^{\prime}|n-1,0| n^{\prime \prime}-1, k_{y}^{\prime}\right)\left[\kappa_{1}^{*} \kappa_{1}^{\prime \prime *}+\kappa_{3}^{*} \kappa_{3}^{\prime \prime *}\right]\right\} e_{z}^{\prime}, \\
\mathcal{D}_{n^{\prime \prime} n^{\prime}}^{-+}(\mathbf{k})= & J\left(k_{x}\left|n^{\prime \prime}-1, k_{y}^{\prime}\right| n^{\prime},-k_{y}^{\prime \prime}\right)\left[\kappa_{4}^{\prime} \kappa_{3}^{\prime \prime}+\kappa_{2}^{\prime} \kappa_{1}^{\prime \prime}\right] e_{-} \\
& +J\left(k_{x}\left|n^{\prime \prime}, k_{y}^{\prime}\right| n^{\prime}-1,-k_{y}^{\prime \prime}\right)\left[\kappa_{3}^{\prime} \kappa_{4}^{\prime \prime}+\kappa_{1}^{\prime} \kappa_{2}^{\prime \prime}\right] e_{+} \\
& -\left\{J\left(k_{x}\left|n^{\prime \prime}, k_{y}^{\prime}\right| n^{\prime},-k_{y}^{\prime \prime}\right)\left[\kappa_{4}^{\prime} \kappa_{4}^{\prime \prime}+\kappa_{2}^{\prime} \kappa_{2}^{\prime \prime}\right]-J\left(k_{x}\left|n^{\prime \prime}-1, k_{y}^{\prime}\right| n^{\prime}-1,-k_{y}^{\prime \prime}\right)\left[\kappa_{3}^{\prime} \kappa_{3}^{\prime \prime}+\kappa_{1}^{\prime} \kappa_{1}^{\prime \prime}\right]\right\} e_{z},
\end{aligned}
$$

where the polarization vector $e_{\mu}=\left(0, e_{x}, e_{y}, e_{z}\right)$ is specified by $e_{ \pm}=e_{x} \pm i e_{y}$ and $e_{z}$, and similarly for the final photon polarizations (primed). The other three $\mathcal{D} \mathrm{s}$ in Eq. (2) are not displayed here for brevity; they can be obtained from those in Eq. (3) simply by the interchange $e_{+} \leftrightarrow e_{-}$of polarization components (and similarly for primed components) and a relabelling of the $J$ s that produces a correspondence $\mathcal{D}_{n^{\prime} n}^{q^{\prime} q}(\mathbf{k}) \rightarrow \mathcal{D}_{n n^{\prime}}^{-q-q^{\prime}}(\mathbf{k})$.

Several notations need to be identified. First, the particles have energies $\varepsilon$, and momentum components $p_{z}$ along the field. The energies $\varepsilon$ and $\varepsilon_{0}$ that appear here are, respectively, with and without the parallel momentum $p_{z}$ :

$$
\varepsilon=\sqrt{1+p_{z}^{2}+2 n B} \quad, \quad \varepsilon_{0}=\sqrt{1+2 n B}
$$

with $n$ denoting the Landau level quantum numbers, as usual. The other quantum number pertaining to the eigenstates of $\mu_{z}$ is $\sigma= \pm 1$, which signifies the spin state of the fermions ( [1] used the label $\tau$; here the notation of Melrose and Parle 15 is preferred), and satisfies $\mu_{z} \psi=\sigma \varepsilon_{0} \psi$. It does not appear explicitly in $\varepsilon$, but is embedded in the spinor coefficients $\kappa_{i}$ :

$$
\left(\begin{array}{c}
\kappa_{1} \\
\kappa_{2} \\
\kappa_{3} \\
\kappa_{4}
\end{array}\right) \equiv\left(\begin{array}{cccc}
\delta_{-} & \delta_{+} & 0 & 0 \\
\delta_{+} & \delta_{-} & 0 & 0 \\
0 & 0 & \delta_{-} & -\delta_{+} \\
0 & 0 & -\delta_{+} & \delta_{-}
\end{array}\right)\left(\begin{array}{c}
\sqrt{\left(\varepsilon_{0}+1\right)\left(\varepsilon+\varepsilon_{0}\right)} \\
-i p_{z} \sqrt{\frac{\varepsilon_{0}-1}{\varepsilon+\varepsilon_{0}}} \\
p_{z \sqrt{\frac{\varepsilon_{0}+1}{\varepsilon+\varepsilon_{0}}}} \\
i \sqrt{\left(\varepsilon_{0}-1\right)\left(\varepsilon+\varepsilon_{0}\right)}
\end{array}\right),
$$

for $\delta_{+}=\delta_{\sigma, 1}$ and $\delta_{-}=\left(1-\delta_{n, 0}\right) \delta_{\sigma,-1}$ where the spin quantum number $\sigma$ takes on two values except for the $n=0$ ground state (zeroth Landau level), where only $\sigma=1$ is permissible. Here $\delta_{i, j}$ is the familiar Kronecker delta. The 
primed coefficients $\kappa_{i}^{\prime}$ and $\kappa_{i}^{\prime \prime}$ are similarly defined in terms of primed momenta and Landau level quantum numbers, subject to the momentum conservation implicit in Eq. (2).

The $J$ functions that appear in Eq. (3) are integrals over the oscillator functions (Hermite polynomial products), a form undeveloped in [1]. Here, Eq. (7.377) of [33] is employed to express these integrals analytically in terms of generalized Laguerre polynomials, $L_{n}^{n^{\prime}-n}(x)$ (see also Eq. (47) of [15]):

$$
J\left(\alpha\left|n^{\prime}, \beta^{\prime}\right| n, \beta\right)=\exp \left(-i \frac{\alpha}{2 B}\left[\beta+\beta^{\prime}\right]\right)\left\{i e^{-i \psi}\right\}^{n^{\prime}-n} I_{n^{\prime}, n}\left(\frac{\rho^{2}}{2 B}\right)
$$

where $\rho(>0)$ and the phase $\psi$ are introduced for convenience of notation: $\alpha=\rho \cos \psi$ and $\beta^{\prime}-\beta=\rho \sin \psi$. Note that the $\beta \mathrm{s}$ are always $k_{y} \mathrm{~s}$ and $\alpha$ is always a $k_{x}$. Here the $I_{n^{\prime}, n}$ functions follow the Sokolov and Ternov convention 29] up to a factor of $n$ !, being related to the $\mathbf{J}$ functions of Melrose and Parle [15], and both are defined in terms of the generalized Laguerre polynomials (see [33]):

$$
I_{n^{\prime}, n}(x)=(-1)^{n^{\prime}-n} I_{n, n^{\prime}}(x)=\mathbf{J}_{n^{\prime}-n}^{n}(x) \equiv \sqrt{\frac{n !}{n^{\prime} !}} e^{-x / 2} x^{\left(n^{\prime}-n\right) / 2} L_{n}^{n^{\prime}-n}(x), \quad n^{\prime}>n .
$$

Values for $n>n^{\prime}$ are obtained by interchanging indices, as indicated. Hereafter, the (modified) Sokolov and Ternov convention for writing the Laguerre polynomials will be adopted. Complex conjugation of Eq. (6) can be used to establish the identity

$$
\mathcal{D}_{n^{\prime} n}^{q^{\prime} q}(\mathbf{k})=(-1)^{n^{\prime}-n}\left\{\mathcal{D}_{n n^{\prime}}^{-q-q^{\prime}}(\mathbf{k})\right\}^{*}
$$

noting that the $\kappa$ products are either purely imaginary or real, for all choices of spin quantum numbers. The three factors like $(-1)^{n^{\prime}-n}$ appearing in the second product of three $\mathcal{D}$ s in Eq. (2) cancel, leading to this product being just the complex conjugate of the first three $\mathcal{D}$ s. This useful symmetry property clearly underlines the convenience of the Sokolov and Ternov choice of wavefunctions when adopting real components for the photon polarization.

The form of the contribution to the S-matrix element in Eq. (2) is identical to that for $S_{f i, 1}^{(3)}$ given in Eq. (25) of Mentzel, Berg and Wunner [1]. In the same fashion, it can be found that the expression derived here for $S_{f i, 2}^{(3)}$ is absolutely identical to Eq. (26) of [1], thereby providing confirmation of their analytic developments; it can be obtained by using the substitutions $\mathbf{k}^{\prime} \leftrightarrow \mathbf{k}^{\prime \prime}, n^{\prime} \leftrightarrow n^{\prime \prime}$ and $e^{\prime} \leftrightarrow e^{\prime \prime}$ in Eq. (3). All other $S_{f i, j}^{(3)}$ contributions result from application of the cyclic permutations

$$
\begin{aligned}
& P_{+1}: k_{\mu}^{\prime \prime}, e_{\mu}^{\prime \prime} \rightarrow k_{\mu}^{\prime}, e_{\mu}^{\prime} \quad, \quad k_{\mu}^{\prime}, e_{\mu}^{\prime} \rightarrow-k_{\mu}, e_{\mu}^{*}, \quad-k_{\mu}, e_{\mu}^{*} \rightarrow k_{\mu}^{\prime \prime}, e_{\mu}^{\prime \prime} ; \\
& P_{-1}:-k_{\mu}, e_{\mu}^{*} \rightarrow k_{\mu}^{\prime}, e_{\mu}^{\prime} \quad, \quad k_{\mu}^{\prime}, e_{\mu}^{\prime} \rightarrow k_{\mu}^{\prime \prime}, e_{\mu}^{\prime \prime}, k_{\mu}^{\prime \prime}, e_{\mu}^{\prime \prime} \rightarrow-k_{\mu}, e_{\mu}^{*},
\end{aligned}
$$

where $k_{\mu}=(\omega, \mathbf{k}), e_{\mu}=\left(0, e_{x}, e_{y}, e_{z}\right)$, etc. Observe also that a minus sign and the complex conjugation of the polarizations are always associated with the initial photon since it is absorbed in the process. Given these permutations, the crossing symmetry for splitting is manifested in the following relationship between the various terms like those in Eq. (2) that contribute to Eq. (11):

$$
S_{f i, 3}^{(3)}=P_{-1} S_{f i, 2}^{(3)}, \quad S_{f i, 4}^{(3)}=P_{+1} S_{f i, 1}^{(3)}, \quad S_{f i, 5}^{(3)}=P_{+1} S_{f i, 2}^{(3)}, \quad S_{f i, 6}^{(3)}=P_{-1} S_{f i, 1}^{(3)},
$$

where the permutations act as operators. This symmetry can be expressed in a multitude of ways using the identities $P_{+1} P_{-1}=I=P_{-1} P_{+1}$ and $P_{ \pm 1}^{3}=I$.

It is important to remark that the derivation of analytic forms by Mentzel, Berg and Wunner is not the first in the literature relating to S-matrix applications to photon splitting. The papers by Melrose and Parle 15, 13, 14, dealing with various aspects of QED in strong magnetic fields, specifically from a wave dispersion/response tensor approach, constructed the S-matrix element for splitting in Eqs. (46) and (47) of [13], which incorporated the quadratic vacuum response tensor given in Eq. (36) of 14. This tensor is obviously of a standard S-matrix Landau representation appearance. Eqs. (2) and (3) can be generated directly (and also $S_{f i, 2}^{(3)}$ ) from the Melrose and Parle evaluation after a modicum of algebra. Hence, Eqs. (2) and (3) here, and Eqs. (25) and (26) can be used as reliable starting points for further S-matrix developments. 


\section{A. Analytic Reduction: Summation over Spin States}

The form in Eqs. (2) and (3) is quite cumbersome. It can be simplified considerably by (i) specializing to specific but representative directions of photon propagation and (ii) analytically performing the summations over spin states $\sigma, \sigma^{\prime}$ and $\sigma^{\prime \prime}$. Restricting the photon motion to the $x$-direction yields photon motion perpendicular to the field: since splitting is collinear in the non-dispersive limit discussed earlier in this paper, it follows that $k_{z}=k_{z}^{\prime}=k_{z}^{\prime \prime}=0$. This choice dramatically simplifies coefficients of the Laguerre polynomials in Eq. (3). Without significant loss of generality, setting $k_{y}=k_{y}^{\prime}=k_{y}^{\prime \prime}=0$ removes nearly all of the phase factors in the definition of the $J \mathrm{~s}$ in Eq. (6), leaving just $i^{n^{\prime}-n}$. Three such factors emerge in the triple product of $\mathcal{D} \mathrm{s}$, leading to a factor of $(-1)^{n^{\prime \prime}-n^{\prime}}$.

The CP symmetry possessed by the splitting process becomes most evident at this point, since it is now simple to derive the CP selection rules. The specification of $k_{y}=k_{y}^{\prime}=k_{y}^{\prime \prime}=0$ and $k_{z}=k_{z}^{\prime}=k_{z}^{\prime \prime}=0$ yields only one possible component of polarization perpendicular to the field, $e_{\perp} \equiv \varepsilon_{y}=-i \varepsilon_{+}=i \varepsilon_{-}$and one conceivable component of polarization parallel to the field, $e_{\|} \equiv \varepsilon_{z}$ (and similarly for primed quantities). The polarization (electric field) vector of the photons is, of course, normal to the photon momentum vector, which automatically spawns the notation for the two possible polarization states: $\perp: \quad e_{\perp}=1, \quad e_{\|}=0$ and $\|: \quad e_{\perp}=0, \quad e_{\|}=1$. From the presence of subtractions in the numerators of the integrands of Eq. (2) together with the complex conjugation property in Eq. (8) and the proportionality of the $\mathcal{D}$ s to factors like $i^{n}-n$, it follows that only terms with an odd number of $e_{\perp}$ factors contribute to $S_{f i, 1}^{(3)}$, i.e. terms proportional to $e_{\|} e_{\|}^{\prime} e_{\perp}^{\prime \prime}, e_{\|} e_{\perp}^{\prime} e_{\|}^{\prime \prime}, e_{\perp} e_{\|}^{\prime} e_{\|}^{\prime \prime}$ and $e_{\perp} e_{\perp}^{\prime} e_{\perp}^{\prime \prime}$. All other terms cancel identically to zero. By virtue of the permutation symmetries in Eq. (9), this is also true for all other $S_{f i, j}^{(3)}$. It is then trivial to deduce the CP selection rules for photon splitting, namely that the only permitted transitions are

$$
\perp \rightarrow \perp \perp \quad, \quad \perp \rightarrow\|\| \quad, \quad\|\rightarrow \perp\| \quad .
$$

The three other splitting transitions all have S-matrix elements that are exactly zero for collinear photon momenta, and hence are forbidden. This technique for $\mathrm{CP}$ selection rule derivation was implemented in [1]. These restrictions are simply consequences of the charge conjugation $(\mathrm{C})$ and parity $(\mathrm{P})$ symmetries of the splitting process, i.e. relating to the transformations $\mathbf{k} \rightarrow-\mathbf{k}$ and $\mathbf{B} \rightarrow-\mathbf{B}$.

The summation over the spin states $\sigma, \sigma^{\prime}$ and $\sigma^{\prime \prime}(= \pm 1)$ produces a dramatic simplification in the appearance of the S-matrix elements. Such spin summations act only on the products of the $\kappa_{i} \mathrm{~s}$ that appear in Eq. (3); the algebra is lengthy but straightforward, being facilitated by pairing $S_{f i, j}^{(3)}$ terms with denominators that differ only in the sign of their photon energies. The total splitting rate in Eq. (1) can be written in the form

$$
R_{e \rightarrow e^{\prime} e^{\prime \prime}}=\frac{\alpha_{\mathrm{f}}^{3}}{2 \pi^{2}} \frac{m c^{2}}{\hbar} \int \frac{d \omega^{\prime}}{\omega^{2}}\left|\mathcal{M}_{e \rightarrow e^{\prime} e^{\prime \prime}}\right|^{2},
$$

where $\omega^{\prime \prime}=\omega-\omega^{\prime}$ is implicitly understood from the conservation of four-momentum. While these rates will be expressed for photon propagation normal to the uniform magnetic field, the results for general photon obliquities $\theta$ to $\mathbf{B}$ can be obtained via a simple Lorentz transformation: $\omega \rightarrow \omega \sin \theta, \omega^{\prime} \rightarrow \omega^{\prime} \sin \theta, \omega^{\prime \prime} \rightarrow \omega^{\prime \prime} \sin \theta$, together with an extra multiplicative factor of $\sin \theta$ applied to the rate in Eq. (12).

The momentum dependence in the integrands of Eq. (2) can be simplified by forming sums of the products of energy denominators. Separating such sums into real and imaginary parts via the representation $\Sigma_{\lambda, \mu}=\Sigma_{\lambda, \mu}^{\mathrm{R}}+i \Sigma_{\lambda, \mu}^{\mathrm{I}}$, leads to the definition

$$
\begin{aligned}
\Sigma_{\lambda, \mu}^{\mathrm{R}} & =\left\{\frac{1}{\left(\varepsilon^{\prime \prime}+\varepsilon+\omega^{\prime}\right)\left(\varepsilon^{\prime}+\varepsilon^{\prime \prime}+\omega\right)}+\frac{1}{\left(\varepsilon^{\prime \prime}+\varepsilon-\omega^{\prime}\right)\left(\varepsilon^{\prime}+\varepsilon^{\prime \prime}-\omega\right)}\right\} \\
& +\lambda\left\{\frac{1}{\left(\varepsilon^{\prime}+\varepsilon^{\prime \prime}-\omega\right)\left(\varepsilon+\varepsilon^{\prime}-\omega^{\prime \prime}\right)}+\frac{1}{\left(\varepsilon^{\prime}+\varepsilon^{\prime \prime}+\omega\right)\left(\varepsilon+\varepsilon^{\prime}+\omega^{\prime \prime}\right)}\right\} \\
& +\mu\left\{\frac{1}{\left(\varepsilon+\varepsilon^{\prime}+\omega^{\prime \prime}\right)\left(\varepsilon^{\prime \prime}+\varepsilon-\omega^{\prime}\right)}+\frac{1}{\left(\varepsilon+\varepsilon^{\prime}-\omega^{\prime \prime}\right)\left(\varepsilon^{\prime \prime}+\varepsilon+\omega\right)}\right\},
\end{aligned}
$$

of the real part, where $\lambda$ and $\mu$ assume the values \pm 1 . The imaginary part is not explicitly stated since it will not be of use in the subsequent developments. The momentum integrations over these $\Sigma_{\lambda, \mu}^{\mathrm{R}}$ then assume one of the forms

$$
\begin{gathered}
\mathcal{I}_{n}=\int_{-\infty}^{\infty} \frac{p_{z}^{2 n} d p_{z}}{\varepsilon \varepsilon^{\prime} \varepsilon^{\prime \prime}} \Sigma_{1,1}^{\mathrm{R}}, \\
\mathcal{J}=\int_{-\infty}^{\infty} \frac{d p_{z}}{\varepsilon} \Sigma_{1,-1}^{\mathrm{R}}, \mathcal{J}^{\prime}=\int_{-\infty}^{\infty} \frac{d p_{z}}{\varepsilon^{\prime}} \Sigma_{-1,1}^{\mathrm{R}}, \mathcal{J}^{\prime \prime}=\int_{-\infty}^{\infty} \frac{d p_{z}}{\varepsilon^{\prime \prime}} \Sigma_{-1,-1}^{\mathrm{R}}
\end{gathered}
$$


for $n=0$ or 1 ; generalizations to complex $\Sigma_{\lambda, \mu}$ (relevant to calculating splitting rates above pair threshold and near pair resonances) are routine. These manipulations yield the following compact forms for the $\mathcal{M}_{e \rightarrow e^{\prime} e^{\prime \prime}}$ coefficients in Eq. (12):

$$
\begin{gathered}
\mathcal{M}_{\perp \rightarrow\|\|}=-\frac{B}{4} \sum_{n, n^{\prime}, n^{\prime \prime}}(-1)^{n^{\prime \prime}-n^{\prime}}\left\{\sqrt{8 n n^{\prime} n^{\prime \prime} B^{3}} \mathcal{I}_{0} \triangle_{1}^{\perp \rightarrow\|\|}+\sqrt{2 n^{\prime \prime} B}\left[\mathcal{J}^{\prime \prime}+\mathcal{I}_{1}-\mathcal{I}_{0}\right] \triangle_{2}^{\perp \rightarrow\|\|}\right. \\
\left.+\sqrt{2 n^{\prime} B}\left[\mathcal{J}^{\prime}-\mathcal{I}_{1}+\mathcal{I}_{0}\right] \triangle_{3}^{\perp \rightarrow\|\|}+\sqrt{2 n B}\left[\mathcal{J}+\mathcal{I}_{1}+\mathcal{I}_{0}\right] \triangle_{4}^{\perp \rightarrow\|\|}\right\} \\
\mathcal{M}_{\perp \rightarrow \perp \perp}=-\frac{B}{4} \sum_{n, n^{\prime}, n^{\prime \prime}}(-1)^{n^{\prime \prime}-n^{\prime}}\left\{\sqrt{8 n n^{\prime} n^{\prime \prime} B^{3}} \mathcal{I}_{0} \triangle_{1}^{\perp \rightarrow \perp \perp}+\sqrt{2 n^{\prime \prime} B}\left[\mathcal{J}^{\prime \prime}-\mathcal{I}_{1}-\mathcal{I}_{0}\right] \triangle_{2}^{\perp \rightarrow \perp \perp}\right. \\
\left.+\sqrt{2 n^{\prime} B}\left[\mathcal{J}^{\prime}+\mathcal{I}_{1}+\mathcal{I}_{0}\right] \triangle_{3}^{\perp \rightarrow \perp \perp}+\sqrt{2 n B}\left[\mathcal{J}+\mathcal{I}_{1}+\mathcal{I}_{0}\right] \triangle_{4}^{\perp \rightarrow \perp \perp}\right\} \\
\mathcal{M}_{\|\rightarrow \perp\|}=-\frac{B}{4} \sum_{n, n^{\prime}, n^{\prime \prime}}(-1)^{n^{\prime \prime}-n^{\prime}}\left\{\sqrt{8 n n^{\prime} n^{\prime \prime} B^{3}} \mathcal{I}_{0} \triangle_{1}^{\|\rightarrow \perp\|}+\sqrt{2 n^{\prime \prime} B}\left[\mathcal{J}^{\prime \prime}+\mathcal{I}_{1}-\mathcal{I}_{0}\right] \triangle_{2}^{\|\rightarrow\|}\right. \\
\left.+\sqrt{2 n^{\prime} B}\left[\mathcal{J}^{\prime}+\mathcal{I}_{1}+\mathcal{I}_{0}\right] \triangle_{3}^{\|\rightarrow \perp\|}+\sqrt{2 n B}\left[\mathcal{J}-\mathcal{I}_{1}+\mathcal{I}_{0}\right] \triangle_{4}^{\|\rightarrow\|}\right\},
\end{gathered}
$$

results that are to be used in conjunction with Eq. (12). The factor of $-B / 4$ is introduced to render the scaled amplitudes positive, and also to afford a direct mapping onto limiting forms obtained [8, 10] by the proper-time technique, as will become evident in Section III. The $\Delta_{i}^{e \rightarrow e^{\prime} e^{\prime \prime}}$ are differences of triple products of generalized

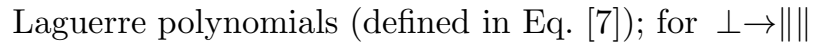

$$
\begin{aligned}
& \triangle_{1}^{\perp \rightarrow\|\|}=I_{n-1, n^{\prime}-1}^{\prime \prime} I_{n^{\prime \prime}, n}^{\prime} I_{n^{\prime}, n^{\prime \prime}-1}-I_{n, n^{\prime}}^{\prime \prime} I_{n^{\prime \prime}-1, n-1}^{\prime} I_{n^{\prime}-1, n^{\prime \prime}} \\
& \triangle_{2}^{\perp \rightarrow\|\|}=I_{n-1, n^{\prime}-1}^{\prime \prime} I_{n^{\prime \prime}-1, n-1}^{\prime} I_{n^{\prime}-1, n^{\prime \prime}}-I_{n, n^{\prime}}^{\prime \prime} I_{n^{\prime \prime}, n}^{\prime} I_{n^{\prime}, n^{\prime \prime}-1} \\
& \triangle_{3}^{\perp \rightarrow\|\|}=I_{n-1, n^{\prime}-1}^{\prime \prime} I_{n^{\prime \prime}-1, n-1}^{\prime \prime} I_{n^{\prime}, n^{\prime \prime}-1}-I_{n, n^{\prime}}^{\prime \prime} I_{n^{\prime \prime}, n}^{\prime} I_{n^{\prime}-1, n^{\prime \prime}} \\
& \triangle_{4}^{\perp \rightarrow\|\|}=I_{n-1, n^{\prime}-1}^{\prime \prime} I_{n^{\prime \prime}, n}^{\prime} I_{n^{\prime}-1, n^{\prime \prime}}-I_{n, n^{\prime}}^{\prime \prime} I_{n^{\prime \prime}-1, n-1}^{\prime} I_{n^{\prime}, n^{\prime \prime}-1},
\end{aligned}
$$

where the Sokolov and Ternov representation of the associated Laguerre functions in Eq. (7) is used together with the priming notation

$$
I_{n^{\prime}, n} \equiv I_{n^{\prime}, n}\left(\frac{\omega^{2}}{2 B}\right) \quad, \quad I_{n^{\prime}, n}^{\prime} \equiv I_{n^{\prime}, n}\left(\frac{\left[\omega^{\prime}\right]^{2}}{2 B}\right) \quad, \quad I_{n^{\prime}, n}^{\prime \prime} \equiv I_{n^{\prime}, n}\left(\frac{\left[\omega^{\prime \prime}\right]^{2}}{2 B}\right)
$$

thereby aiding brevity. For the $\perp \rightarrow \perp \perp$ mode,

$$
\begin{aligned}
& \triangle_{1}^{\perp \rightarrow \perp \perp}=I_{n, n^{\prime}-1}^{\prime \prime} I_{n^{\prime \prime}, n-1}^{\prime} I_{n^{\prime}, n^{\prime \prime}-1}-I_{n-1, n^{\prime}}^{\prime \prime} I_{n^{\prime \prime}-1, n}^{\prime} I_{n^{\prime}-1, n^{\prime \prime}} \\
& \triangle_{2}^{\perp \rightarrow \perp \perp}=I_{n, n^{\prime}-1}^{\prime \prime} I_{n^{\prime \prime}-1, n}^{\prime} I_{n^{\prime}-1, n^{\prime \prime}}-I_{n-1, n^{\prime}}^{\prime \prime} I_{n^{\prime \prime}, n-1}^{\prime \prime} I_{n^{\prime}, n^{\prime \prime}-1} \\
& \triangle_{3}^{\perp \rightarrow \perp \perp}=I_{n, n^{\prime}-1}^{\prime \prime} I_{n^{\prime \prime}-1, n}^{\prime} I_{n^{\prime}, n^{\prime \prime}-1}-I_{n-1, n^{\prime}}^{\prime \prime} I_{n^{\prime \prime}, n-1}^{\prime} I_{n^{\prime}-1, n^{\prime \prime}} \\
& \triangle_{4}^{\perp \rightarrow \perp \perp}=I_{n, n^{\prime}-1}^{\prime \prime} I_{n^{\prime \prime}, n-1}^{\prime} I_{n^{\prime}-1, n^{\prime \prime}}-I_{n-1, n^{\prime}}^{\prime \prime} I_{n^{\prime \prime}-1, n}^{\prime} I_{n^{\prime}, n^{\prime \prime}-1}
\end{aligned}
$$

and the results for the $\|\rightarrow \perp\|$ mode are not explicitly stated since they can be obtained by exploiting crossing symmetries: the inverse of the permutation in Eq. (19) yields the transformation $\triangle_{1}^{\perp \rightarrow\|\|} \rightarrow-\triangle_{1}^{\|\rightarrow \perp\|}, \triangle_{2}^{\perp \rightarrow\|\|} \rightarrow$ $\triangle_{3}^{\|\rightarrow \perp\|}, \triangle_{3}^{\perp \rightarrow\|\|} \rightarrow-\triangle_{4}^{\|\rightarrow \perp\|}, \triangle_{4}^{\perp \rightarrow\|\|} \rightarrow \triangle_{2}^{\|\rightarrow \perp\|}$. The $\triangle_{i}^{e \rightarrow e^{\prime} e^{\prime \prime}}$ can alternatively be expressed using the $\mathbf{J}_{b}^{a}$ functions of Melrose and Parle as in [11]. Note that the potential subtlety of having to include factors of $1 / 2$ for some contributions from ground intermediate states is eliminated by the specific choice of the Sokolov and Ternov wavefunctions.

The comparative simplicity of the reduced form of the S-matrix element relative to Eq. (2) is both notable and comforting. Unlike Eqs. (25) and (26) of [1], this developed form of the splitting S-matrix element has an appearance 
familiar to S-matrix applications of QED in the Landau representation to strongly-magnetized systems, with products of generalized Laguerre polynomials multiplied by simple combinations of energies and momentum components. Examples of previous work bearing such familiar forms focus largely on lower-order QED processes and include studies of synchrotron radiation [29] 30], single photon pair creation [17,29], and vacuum [34] and plasma [35] polarization.

For the purposes of the analysis in the next section, it is pertinent to define the cyclic permutations

$$
\begin{aligned}
& \omega \rightarrow-\omega^{\prime \prime}, \quad \omega^{\prime} \rightarrow-\omega, \quad \omega^{\prime \prime} \rightarrow \omega^{\prime}, \\
& n \rightarrow n^{\prime \prime}, \quad n^{\prime} \rightarrow n, \quad n^{\prime \prime} \rightarrow n^{\prime},
\end{aligned}
$$

in the spirit of the $P_{+1}$ permutation in Eq. (9). These permutations will appear repeatedly in the developments below, and lead to the following transformation properties of Eq. (13):

$$
\Sigma_{-1,-1}^{\mathrm{R}} \rightarrow-\Sigma_{-1,1}^{\mathrm{R}}, \quad \Sigma_{-1,1}^{\mathrm{R}} \rightarrow \Sigma_{1,-1}^{\mathrm{R}}, \quad \Sigma_{1,-1}^{\mathrm{R}} \rightarrow-\Sigma_{-1,-1}^{\mathrm{R}},
$$

with $\Sigma_{1,1}^{\mathrm{R}}$ being invariant, symmetries that are consequences of the arrangements of electron and positron propagators in the Feynman diagram for splitting. These translate into obvious mappings between $\mathcal{J}$, $\mathcal{J}^{\prime}$ and $\mathcal{J}^{\prime \prime}$ and an invariance of the $I_{n}$. It is also easily seen that under this cyclic permutation, the factor in braces in the summation for $\mathcal{M}_{\perp \rightarrow \perp \perp}$ is invariant, while the equivalent factor in the summation for $\mathcal{M}_{\|\rightarrow \perp\|}$ maps over (up to a minus sign) to the factor in braces in the $\mathcal{M}_{\perp \rightarrow\|\|}$ summation. As will become evident in Section III, the remaining powers of -1 in the summations do not provide any unsatisfactory interference in the limits of low photon energy $(\omega \ll 1)$ and high fields $(B \gg 1)$, so that permutation symmetry can be extended to the total amplitudes in these specific parameter regimes.

\section{B. Analytic Reduction: Integration over Parallel Momentum}

Further analytic development is not only possible, but also desirable, given that the integrations over the momentum $p_{z}$ parallel to the field can be expressed compactly in terms of elementary functions. Such tractability facilitates both numerical evaluations and the derivation of asymptotic limits. In proceeding, since results are sought at energies sufficiently remote from pair creation resonances, the imaginary parts of the denominators in the $\Sigma_{\lambda, \mu}$ are dropped in all further considerations, i.e., we consider only the functions $\Sigma_{\lambda, \mu}^{\mathrm{R}}=\operatorname{Re} \Sigma_{\lambda, \mu}$.

It turns out that carefully-constructed contour integrations in the complex $p_{z}$ plane do not facilitate the $p_{z}$ integrations. Hence the first step in integrating over $p_{z}$ is effected by the more cumbersome and less elegant approach of completing the squares and rationalizing the denominators using products of factors like $\left(\varepsilon^{\prime} \pm \varepsilon^{\prime \prime} \pm \omega\right)$. These factors define poles $p_{i j}$ of the $p_{z}$ integration for $i$ and $j$ being some combination of $n, n^{\prime}$ and $n^{\prime \prime}$. Such poles fall into two types: pair creation ones (e.g. see [17]) that contribute only above pair threshold, due to the structure of the splitting rate, and cyclotronic ones that must be considered below pair threshold. The appearance of such cyclotronic poles is an artifact of the rationalization of denominators, so that they are really pseudo-poles of the subsequent analysis; a consistency check on the algebra is that the S-matrix element be effectively continuous across them. It is convenient to define energies that correspond to the $p_{i j}$ poles:

$$
\varepsilon_{n n^{\prime}}=\frac{\left(\omega^{\prime \prime}\right)^{2}+\mathcal{N}-\mathcal{N}^{\prime}}{2 \omega^{\prime \prime}} \quad, \quad \varepsilon_{n^{\prime} n^{\prime \prime}}=-\frac{\omega^{2}+\mathcal{N}^{\prime}-\mathcal{N}^{\prime \prime}}{2 \omega} \quad, \quad \varepsilon_{n^{\prime \prime} n}=\frac{\left(\omega^{\prime}\right)^{2}+\mathcal{N}^{\prime \prime}-\mathcal{N}}{2 \omega^{\prime}}
$$

and three others paired with these, which are obtained via the relations $\varepsilon_{n^{\prime} n}+\varepsilon_{n n^{\prime}}=\omega^{\prime \prime}, \varepsilon_{n^{\prime \prime} n^{\prime}}+\varepsilon_{n^{\prime} n^{\prime \prime}}=-\omega$ and $\varepsilon_{n n^{\prime \prime}}+\varepsilon_{n^{\prime \prime} n}=\omega^{\prime}$. Here the notation

$$
\mathcal{N}=1+2 n B, \quad \mathcal{N}^{\prime}=1+2 n^{\prime} B, \quad \mathcal{N}^{\prime \prime}=1+2 n^{\prime \prime} B
$$

is used for the purposes of abbreviation. Observe that, taking advantage of the subjectivity of such definitions, a minus sign appears in front of the expression for $\varepsilon_{n^{\prime} n^{\prime \prime}}$, a choice that preserves symmetries induced by the mapping in Eq. (19) in the results that follow. These definitions spawn the following useful identities for the momentum poles:

$$
\begin{aligned}
p_{n n^{\prime}}^{2} & =\varepsilon_{n n^{\prime}}^{2}-\mathcal{N}=\varepsilon_{n^{\prime} n}^{2}-\mathcal{N}^{\prime} \\
p_{n^{\prime} n^{\prime \prime}}^{2} & =\varepsilon_{n^{\prime} n^{\prime \prime}}^{2}-\mathcal{N}^{\prime}=\varepsilon_{n^{\prime \prime} n^{\prime}}^{2}-\mathcal{N}^{\prime \prime} \\
p_{n^{\prime \prime} n}^{2} & =\varepsilon_{n^{\prime \prime} n}^{2}-\mathcal{N}^{\prime \prime}=\varepsilon_{n n^{\prime \prime}}^{2}-\mathcal{N},
\end{aligned}
$$

which immediately imply the possibility of poles along the imaginary axis. In fact, $p_{n n^{\prime}}^{2} \geq-\min \left\{\mathcal{N}, \mathcal{N}^{\prime}\right\}$, with equality for $\omega^{\prime \prime}=\left|\mathcal{N}-\mathcal{N}^{\prime}\right|^{1 / 2}$, and likewise for the other poles. Note that for the one-vertex calculations of cyclotron 
emission and single photon pair creation and annihilation, the requirement that such poles be real, corresponding to real components of particle momenta on external lines, is precisely what generates thresholds (e.g. [17]) and kinematic cutoffs (e.g. [30]) for transitions involving various states.

The rationalization of the denominators yields relatively compact decompositions for these sums, after much cancellation and simplification. They take the form

$$
\Sigma_{\lambda, \mu}^{\mathrm{R}}=c_{\lambda, \mu}+\frac{2}{W}\left\{t_{\lambda, \mu}^{\varepsilon \varepsilon^{\prime}} \varepsilon \varepsilon^{\prime}+t_{\lambda, \mu}^{\varepsilon^{\prime} \varepsilon^{\prime \prime}} \varepsilon^{\prime} \varepsilon^{\prime \prime}+t_{\lambda, \mu}^{\varepsilon^{\prime \prime} \varepsilon} \varepsilon^{\prime \prime} \varepsilon\right\}
$$

the simplicity of which is contingent upon the energy-conservation restriction $\omega^{\prime \prime}=\omega-\omega^{\prime}$. Here

$$
W=\omega \omega^{\prime} \omega^{\prime \prime}+\omega \mathcal{N}-\omega^{\prime} \mathcal{N}^{\prime}-\omega^{\prime \prime} \mathcal{N}^{\prime} .
$$

Identities such as $W=-2 \omega \omega^{\prime}\left(\varepsilon_{n^{\prime \prime} n^{\prime}}+\varepsilon_{n^{\prime \prime} n}\right)$ prove useful in the ensuing analysis. The $c_{\lambda, \mu}$ and $t_{\lambda, \mu}$ coefficients assume simple forms when expressed as partial fractions. Consider first the result for $\Sigma_{1,1}^{\mathrm{R}}$, which has the coefficients

$$
\begin{aligned}
c_{1,1} & =0 \\
t_{1,1}^{\varepsilon \varepsilon^{\prime}} & =\frac{\varepsilon_{n^{\prime \prime} n}}{p_{n^{\prime \prime} n}^{2}-p_{z}^{2}}+\frac{\varepsilon_{n^{\prime \prime} n^{\prime}}}{p_{n^{\prime} n^{\prime \prime}}^{2}-p_{z}^{2}} \\
t_{1,1}^{\varepsilon^{\prime} \varepsilon^{\prime \prime}} & =\frac{\varepsilon_{n n^{\prime \prime}}}{p_{n^{\prime \prime} n}^{2}-p_{z}^{2}}+\frac{\varepsilon_{n n^{\prime}}}{p_{n n^{\prime}}^{2}-p_{z}^{2}} \\
t_{1,1}^{\varepsilon^{\prime \prime} \varepsilon} & =\frac{\varepsilon_{n^{\prime} n}}{p_{n n^{\prime}}^{2}-p_{z}^{2}}+\frac{\varepsilon_{n^{\prime} n^{\prime \prime}}}{p_{n^{\prime} n^{\prime \prime}}^{2}-p_{z}^{2}} .
\end{aligned}
$$

Observe that a cyclic symmetry is immediately apparent: $\Sigma_{1,1}^{\mathrm{R}}$ is invariant under the permutation in Eq. (19), as is evident from its original definition in Eq. (13). Similarly, the algebraic developments yield coefficients for the $\Sigma_{-1,-1}^{\mathrm{R}}$ sum, which appears in the $\varepsilon \varepsilon^{\prime} \Delta_{2}$ terms, as

$$
\begin{aligned}
& c_{-1,-1}=\frac{1}{\omega \omega}-\frac{2}{W}\left\{\frac{\varepsilon_{n^{\prime \prime} n^{\prime}} \varepsilon_{n^{\prime} n^{\prime \prime}}}{p_{n^{\prime} n^{\prime \prime}}^{2}-p_{z}^{2}}\left[\varepsilon_{n^{\prime \prime} n^{\prime}}+\omega^{\prime}\right]+\frac{\varepsilon_{n^{\prime \prime} n} \varepsilon_{n n^{\prime \prime}}}{p_{n^{\prime \prime} n}^{2}-p_{z}^{2}}\left[\varepsilon_{n^{\prime \prime} n}-\omega\right]\right\} \\
& t_{-1,-1}^{\varepsilon \varepsilon^{\prime}}=0 \\
& t_{-1,-1}^{\varepsilon^{\prime} \varepsilon^{\prime \prime}}=-\frac{\varepsilon_{n^{\prime \prime} n^{\prime}}+\omega^{\prime}}{p_{n^{\prime} n^{\prime \prime}}^{2}-p_{z}^{2}}-\frac{\varepsilon_{n n^{\prime}}}{p_{n n^{\prime}}^{2}-p_{z}^{2}} \\
& t_{-1,-1}^{\varepsilon^{\prime \prime} \varepsilon}=-\frac{\varepsilon_{n^{\prime \prime} n}-\omega}{p_{n^{\prime \prime} n}^{2}-p_{z}^{2}}-\frac{\varepsilon_{n^{\prime} n}}{p_{n n^{\prime}}^{2}-p_{z}^{2}} .
\end{aligned}
$$

The coefficients for the sum $\Sigma_{-1,1}^{\mathrm{R}}$ that appears in the $\varepsilon \varepsilon^{\prime \prime} \Delta_{3}$ terms and the coefficients for the sum $\Sigma_{1,-1}^{\mathrm{R}}$ that appears in the $\varepsilon^{\prime} \varepsilon^{\prime \prime} \Delta_{4}$ terms are similar: there is little need to state them explicitly, since the coefficients possess a relationship to each other due to the permutation symmetry enunciated in Eq. (20).

Given these decompositions, it is now fairly straightforward to evaluate the integrations over $p_{z}$, expressing them in terms of the an elementary function $f$ with real arguments $\varepsilon_{i j}$ :

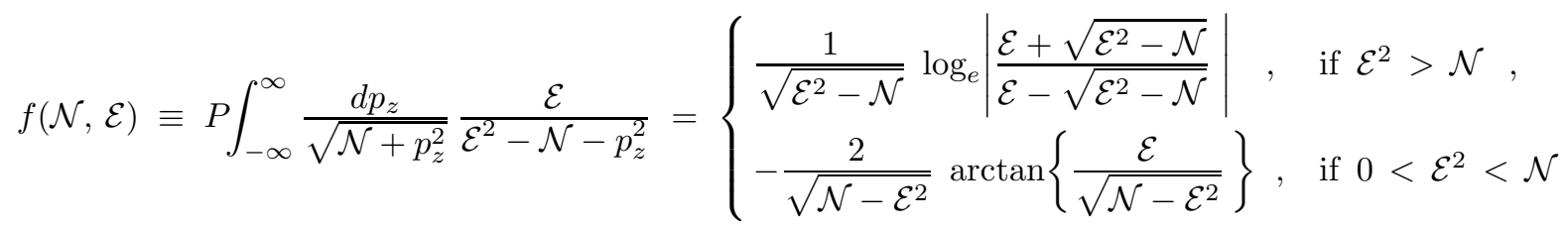

for real $\mathcal{E}$. The identity $\arctan z=(1 / 2 i) \log _{e}[(1+i z) /(1-i z)]$ with $z=-\mathcal{E} / \sqrt{\mathcal{N}-\mathcal{E}^{2}}$ has been used to map across the singularities at $\mathcal{E}= \pm \sqrt{\mathcal{N}}$ (cyclotronic below pair threshold) and guarantee bounded and continuous behaviour of $f(\mathcal{N}, \mathcal{E}) / \mathcal{E}$ at $\mathcal{E}=0$. The integral identity in Eq. (28) can be established quickly with the aid of result 3.513 .2 in [33], using the substitution $p_{z}=\sqrt{\mathcal{N}} \sinh t$ and partial fractions. Note that real values (either positive or negative) of $\mathcal{E}$ are guaranteed by the formalism here, with $\mathcal{E}=0$ being improbable due to the discreteness of the quantum numbers $n, n^{\prime}$ and $n^{\prime \prime}$.

The integration of the coefficients $\mathcal{I}_{0}$ of the $\Delta_{1}$ terms for each of the polarization modes are then straightforward, and the identities in Eq. (23) can be used to advantage. Similar terms appear in the $\mathcal{I}_{1}$ integrations of parts of the 
coefficients of the other $\Delta_{i}$ terms, which also possess integrands with terms proportional to $1 / \varepsilon, 1 / \varepsilon^{\prime}$ and $1 / \varepsilon^{\prime \prime}$ that formally lead to divergences that cancel each other (an artifice introduced by the rationalization of the denominators). Using partial fractions, the divergent contributions can be written as integrals over the finite range $-p \leq p_{z} \leq p$, rearranging to subtract off exactly-cancelling terms, and then taking the limit as $p \rightarrow \infty$. Similar manipulations are used for the $\mathcal{J}^{\prime \prime}$ integration over $\Sigma_{-1,-1}^{\mathrm{R}}$, where again the leading order terms are individually divergent yet collectively convergent. Partial fractions can again be used to enable rearrangements and separate the divergent terms, which are then integrated over finite ranges as with the $\mathcal{I}_{1}$ evaluation. The results are encapsulated in the identities

$$
\begin{aligned}
\mathcal{I}_{0} & =\frac{2}{W}\left\{\mathcal{F}_{n n^{\prime}}+\mathcal{F}_{n^{\prime} n^{\prime \prime}}+\mathcal{F}_{n^{\prime \prime} n}\right\}, \\
\mathcal{I}_{1} & =\mathcal{L}+\frac{2}{W}\left\{p_{n n^{\prime}}^{2} \mathcal{F}_{n n^{\prime}}+p_{n^{\prime} n^{\prime \prime}}^{2} \mathcal{F}_{n^{\prime} n^{\prime \prime}}+p_{n^{\prime \prime} n}^{2} \mathcal{F}_{n^{\prime \prime} n}\right\}, \\
\mathcal{J}^{\prime \prime} & =\mathcal{L}-\frac{2}{W}\left\{\varepsilon_{n n^{\prime}} \varepsilon_{n^{\prime} n} \mathcal{F}_{n n^{\prime}}+\varepsilon_{n^{\prime} n^{\prime \prime}}\left(\varepsilon_{n^{\prime \prime} n^{\prime}}+\omega^{\prime}\right) \mathcal{F}_{n^{\prime} n^{\prime \prime}}+\varepsilon_{n n^{\prime \prime}}\left(\varepsilon_{n^{\prime \prime} n}-\omega\right) \mathcal{F}_{n^{\prime \prime} n}\right\},
\end{aligned}
$$

where

$$
\mathcal{F}_{n n^{\prime}}=f\left(\mathcal{N}, \varepsilon_{n n^{\prime}}\right)+f\left(\mathcal{N}^{\prime}, \varepsilon_{n^{\prime} n}\right), \quad \mathcal{F}_{n^{\prime} n^{\prime \prime}}=f\left(\mathcal{N}^{\prime}, \varepsilon_{n^{\prime} n^{\prime \prime}}\right)+f\left(\mathcal{N}^{\prime \prime}, \varepsilon_{n^{\prime \prime} n^{\prime}}\right), \quad \mathcal{F}_{n^{\prime \prime} n}=f\left(\mathcal{N}^{\prime \prime}, \varepsilon_{n^{\prime \prime} n}\right)+f\left(\mathcal{N}, \varepsilon_{n n^{\prime \prime}}\right),
$$

and

$$
\mathcal{L}=\frac{1}{\omega^{\prime} \omega^{\prime \prime}} \log _{e} \mathcal{N}-\frac{1}{\omega^{\prime \prime} \omega} \log _{e} \mathcal{N}^{\prime}-\frac{1}{\omega \omega^{\prime}} \log _{e} \mathcal{N}^{\prime \prime}
$$

No further integration is necessary: the cyclic permutations in Eq. (19) can be used to quickly derive expressions for $\mathcal{J}^{\prime}$ and $\mathcal{J}$ from Eq. (29).

At this point, it is salient to remark that the divergences at $\mathcal{E}^{2}=\mathcal{N}$ in the functions $f(\mathcal{N}, \mathcal{E})$ pose no problem for the integral evaluations in Eqs. (29), because these functions always appear two at a time. Below the pair threshold, these divergences are cyclotronic in nature, being encountered when $\omega \rightarrow\left|\sqrt{\mathcal{N}^{\prime}}-\sqrt{\mathcal{N}^{\prime \prime}}\right|$ or for similar circumstances for the other photon energies. As $\omega$ tends to such a limit, for example, we observe that $\varepsilon_{n^{\prime} n^{\prime \prime}} \rightarrow \sqrt{\mathcal{N}^{\prime}}$ and $\varepsilon_{n^{\prime \prime} n^{\prime}} \rightarrow-\sqrt{\mathcal{N}^{\prime \prime}}$ when $\mathcal{N}^{\prime}>\mathcal{N}^{\prime \prime}$ (without loss of generality). This opposition of signs guarantees cancellation of divergences when the $\arctan$ form of $f(\mathcal{N}, \mathcal{E})$ is used $(\arctan (1 / z) \rightarrow \pi / 2-z$ as $z \rightarrow 0)$, so that continuity across cyclotron "pseudo-resonances" emerges naturally from Eq. (29), consistent with the continuity of the $\Sigma_{\lambda, \mu}^{\mathrm{R}}$ functions. Continuity across pair resonances does not arise above pair threshold, so that true divergences emerge.

The incorporation of Eq. (29) into the scaled matrix elements in Eq. (15) constitutes the final product of the general analytic developments in this paper, providing rates valid for all energies below pair threshold (and applicable for non-resonant energies above threshold), and for photon propagation normal to the uniform magnetic field. They are eminently suitable for numerical computations, having improved upon the analytic formalism of Mentzel, Berg and Wunner [1] (i.e. Eq. [2) by performing the summations of the spin states and integration over the momenta parallel to the field that are associated with the electron propagators. Such developments are prudent prior to numerical evaluations due to the large degree of cancellation in these sums and integrations.

\section{ASYMPTOTIC LIMITS FOR HIGH $B$ OR SMALL $\omega$}

A fruitful extension of this analysis is the exploration of the simplification of the scattering amplitudes and rates in two particular asymptotic regimes, namely the limit of highly supercritical fields, $B \gg 1$, and the specialization to photon energies well below threshold, i.e. $\omega \ll 1$. The benefits of such an investigation are twofold. First, it provides the first unequivocal analytic demonstration of the equivalence of splitting results from the S-matrix formulation in the Landau representation and effective Lagrangian/proper-time results from Schwinger-type formalisms in well-defined parameter regimes. In doing so, it serves as a powerful check on the developments here. Second, in the $\omega \ll 1$ case, it identifies a new, satisfyingly compact representation of the scattering amplitudes in terms of special functions that leads to an efficient means of computation.

These two parameter regimes are encompassed under the single limit $\omega^{2} \ll 1+2 B$, which thereby identifies the appropriate series expansion of the generalized Laguerre polynomials that appear in the amplitudes. For small arguments $x$, the leading order terms in the series for $I_{n^{\prime}, n}(x)$ can be found in the Appendix of 15 . Given that $n$, 
$n^{\prime}$ and $n^{\prime \prime}$ cluster in a manner such that $\left|n^{\prime}-n\right| \sim\left|n^{\prime \prime}-n\right| \sim 1$, this series converges rapidly provided $n x \ll 1$. Hence $n \omega^{2} /(2 B)$ actually represents the true expansion parameter here, with $\omega^{\prime}$ and $\omega^{\prime \prime}$ being similarly bounded. The leading order terms of such expansions for the $\Delta_{i}^{e \rightarrow e^{\prime} e^{\prime \prime}}$ are linear in the photon energies, while the next higher order terms are cubic; a more detailed exposition can be found in Weise, Baring and Melrose 111. The series for the integrations of $p_{z}$, namely $\mathcal{I}_{0}, \mathcal{I}_{1}, \mathcal{J}^{\prime \prime}, \mathcal{J}^{\prime}$ and $\mathcal{J}$ (which do not depend on the polarization mode) are expansions in $\omega^{2} /(1+2 B)$ rather than $\omega^{2} /(2 B)$. They are independent of photon energy to leading order, with a quadratic scaling with energy to next order. The series for $\omega^{2} \ll 1+2 B$ possess logarithmic character in the quantum numbers in situations when no two of them are equal (i.e. $\mathcal{N} \neq \mathcal{N}^{\prime} \neq \mathcal{N}^{\prime \prime} \neq \mathcal{N}$ ):

$$
\begin{gathered}
\mathcal{I}_{0} \approx \frac{4 \log _{e} \mathcal{N}}{\left(\mathcal{N}-\mathcal{N}^{\prime}\right)\left(\mathcal{N}^{\prime \prime}-\mathcal{N}\right)}+\frac{4 \log _{e} \mathcal{N}^{\prime}}{\left(\mathcal{N}^{\prime}-\mathcal{N}^{\prime \prime}\right)\left(\mathcal{N}-\mathcal{N}^{\prime}\right)}+\frac{4 \log _{e} \mathcal{N}^{\prime \prime}}{\left(\mathcal{N}^{\prime \prime}-\mathcal{N}\right)\left(\mathcal{N}^{\prime}-\mathcal{N}^{\prime \prime}\right)} \\
\mathcal{I}_{1} \approx-\mathcal{J}^{\prime \prime} \approx-\frac{2 \mathcal{N} \log _{e} \mathcal{N}}{\left(\mathcal{N}-\mathcal{N}^{\prime}\right)\left(\mathcal{N}^{\prime \prime}-\mathcal{N}\right)}-\frac{2 \mathcal{N}^{\prime} \log _{e} \mathcal{N}^{\prime}}{\left(\mathcal{N}^{\prime}-\mathcal{N}^{\prime \prime}\right)\left(\mathcal{N}-\mathcal{N}^{\prime}\right)}-\frac{2 \mathcal{N}^{\prime \prime} \log _{e} \mathcal{N}^{\prime \prime}}{\left(\mathcal{N}^{\prime \prime}-\mathcal{N}\right)\left(\mathcal{N}^{\prime}-\mathcal{N}^{\prime \prime}\right)}
\end{gathered}
$$

and additionally involve inverse trigonometric functions when two $n \mathrm{~s}$ (e.g. for $\mathcal{N}=\mathcal{N}^{\prime}$ ) are in fact equal:

$$
\begin{aligned}
& \mathcal{I}_{0} \approx \frac{4}{\left(\mathcal{N}-\mathcal{N}^{\prime \prime}\right)^{2}} \log _{e} \frac{\mathcal{N}}{\mathcal{N}^{\prime \prime}}-\frac{4}{\mathcal{N}\left(\mathcal{N}-\mathcal{N}^{\prime \prime}\right)} Q\left(\frac{\omega^{\prime \prime}}{2 \sqrt{\mathcal{N}}}\right), \\
& \mathcal{I}_{1} \approx-\frac{2 \mathcal{N}^{\prime \prime}}{\left(\mathcal{N}-\mathcal{N}^{\prime \prime}\right)^{2}} \log _{e} \frac{\mathcal{N}}{\mathcal{N}^{\prime \prime}}-\frac{2}{\mathcal{N}-\mathcal{N}^{\prime \prime}}+\frac{4 \mathcal{N}-\left(\omega^{\prime \prime}\right)^{2}}{\mathcal{N}\left(\mathcal{N}-\mathcal{N}^{\prime \prime}\right)} Q\left(\frac{\omega^{\prime \prime}}{2 \sqrt{\mathcal{N}}}\right) \approx \frac{4}{\left(\mathcal{N}-\mathcal{N}^{\prime \prime}\right)} Q\left(\frac{\omega^{\prime \prime}}{2 \sqrt{\mathcal{N}}}\right)-\mathcal{J}^{\prime \prime},
\end{aligned}
$$

where

$$
Q(x)=\frac{\arcsin x}{x \sqrt{1-x^{2}}}
$$

and the identity $\arcsin x=\arctan \left[x / \sqrt{1-x^{2}}\right]$ has been invoked. This retention of the inverse trigonometric functions is particularly relevant for determining the high $B$ limiting forms of the scattering amplitudes. Relations similar to Eq. (33) exist for $\mathcal{N}=\mathcal{N}^{\prime}$ and $\mathcal{N}^{\prime}=\mathcal{N}^{\prime \prime}$, obtained by the cyclic permutations through $\mathcal{N}$ s and photon energies. The lengthier higher order (quadratic) terms are not explicitly stated for the sake of brevity. This concludes the preamble that guides the reader in the subsequent specializations.

\section{A. The Special Case of $B \gg 1$}

This regime is of particular relevance to the study of magnetars such as soft gamma repeaters. For the two modes $\perp \rightarrow\|\|$ and $\|\rightarrow \perp\|$, only the leading order terms for the $\Delta_{i}$ and the momentum integrals presented in Eqs. (32) and (33) are required. Consider first the reduction of $\mathcal{M}_{\perp \rightarrow\|\| \|}$. Here the $\Delta_{2}$ and $\Delta_{3}$ terms contribute leading order terms only through $n^{\prime \prime}=1, n=n^{\prime}=0$ and $n^{\prime}=1, n=n^{\prime \prime}=0$ cases, respectively, where it is necessary to use the full forms in Equation (33), and inverse trigonometric functions appear through the $Q(x)$ function, which assumes the arguments $x=\omega^{\prime} / 2$ and $x=\omega^{\prime \prime} / 2$. A similar $n=1, n^{\prime}=n^{\prime \prime}=0$ term is identically equal to zero by virtue of the $\Delta_{4}$ factor. The contributions from the $\Delta_{1}$ and $\Delta_{4}$ terms possess an entirely different character, being infinite summations over $n$, with the values of $n^{\prime}$ and $n^{\prime \prime}$ being constrained by $\left|n^{\prime}-n\right|+\left|n^{\prime \prime}-n\right| \leq 1$, producing five groupings of the indices. The series is evaluated by truncating the sum at $n \leq k$, relabelling one of the logarithmic terms, and then taking the limit $k \rightarrow \infty$. The net result is (for $\omega<2$ )

$$
\mathcal{M}_{\perp \rightarrow\|\|} \approx \frac{4 \omega^{\prime}}{\omega^{\prime \prime} \sqrt{4-\left(\omega^{\prime \prime}\right)^{2}}} \arcsin \left(\frac{\omega^{\prime \prime}}{2}\right)+\frac{4 \omega^{\prime \prime}}{\omega^{\prime} \sqrt{4-\left(\omega^{\prime}\right)^{2}}} \arcsin \left(\frac{\omega^{\prime}}{2}\right)-\omega \quad, \quad B \gg 1,
$$

which, when combined with Equation (12), yields the asymptotic high-B result derived by Baier et al. [8], and reproduced independently by Baring \& Harding [10]; the overall rate for $\perp \rightarrow\|\| \|$ approaches a value independent of $B$. Observe that the manifestations of the pair creation threshold for each of the final photons of $\|$ polarization (i.e. at $\omega^{\prime}=2$ and $\omega^{\prime \prime}=2$ ) are the individually-divergent coefficients of the inverse trigonometric functions. Yet, collectively, due to the energy conservation relation $\omega=\omega^{\prime}+\omega^{\prime \prime}$, such divergences cancel each other to yield a finite overall result as $\omega \rightarrow 2$. For the incident photon of $\perp$ polarization, the pair threshold of $1+\sqrt{1+2 B}$ is remote from $\omega=2$ so that it would only become explicitly apparent when the amplitude was evaluated to higher order in $B$. Note also that the $\omega \ll 1$ limit of Eq. (35) is $\omega \omega^{\prime} \omega^{\prime \prime} / 6$ and reproduces results obtained in [1] and [6]. The functional form of Eq. (35) is plotted in Figure 1 . 

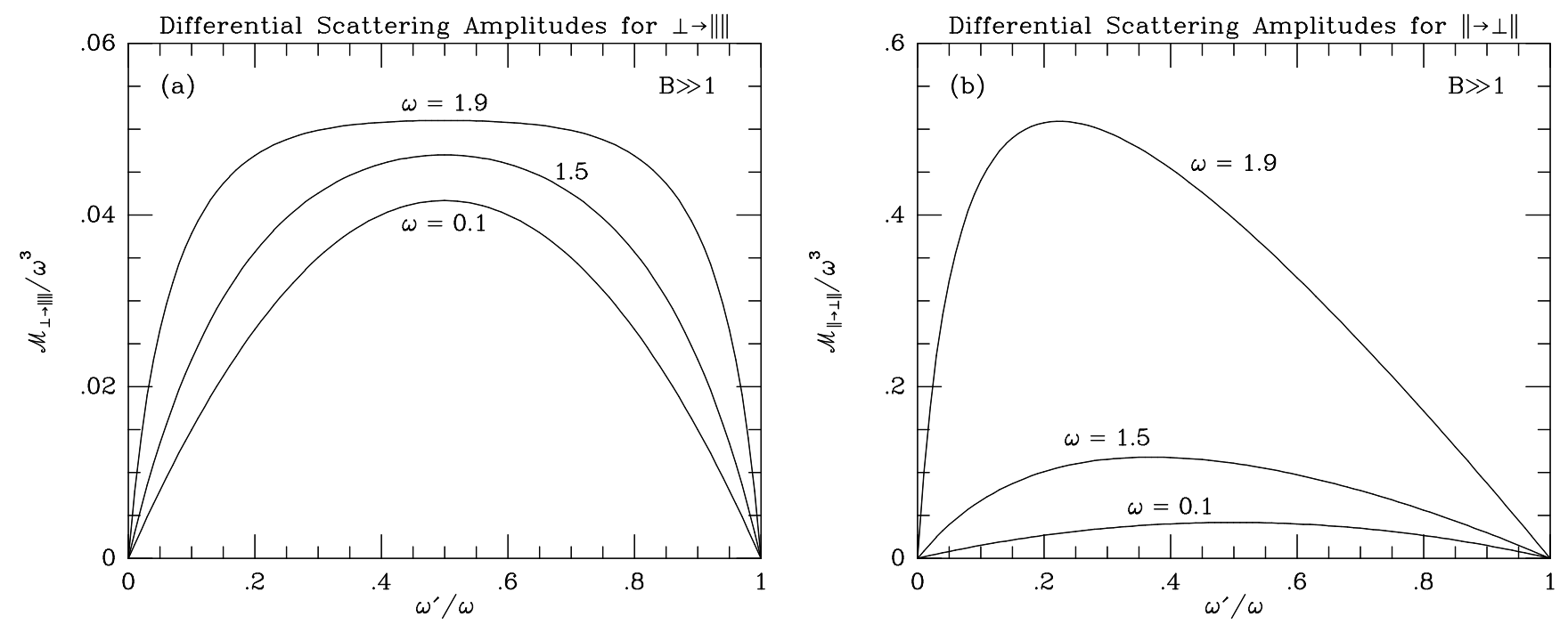

FIG. 1. The dependence of the scattering amplitudes for $B \gg 1$, scaled by $\omega^{3}$, on the fractional energy $\omega^{\prime} / \omega$ of one of the produced photons, for three different incident photon energies $\omega$ (in units of $m c^{2}$ ), as labelled. Only the two polarization modes with amplitudes asymptotically independent of $B$ (in units of $B_{\mathrm{c}}$ ) in this ultra-quantum limit are depicted, namely (a) $\perp \rightarrow\|\|$ and (b) $\|\rightarrow \perp\|$; their functional forms are given in Eqs. (35) and (36), respectively. The shape of the amplitude curves for $\perp \rightarrow \perp \perp$ is independent of $\omega$ and is very close to that of the $\omega=0.1$ curves in panels (a) and (b). While the $\perp \rightarrow\|\|$ curves are necessarily symmetric about $\omega^{\prime}=\omega / 2$, asymmetry is present in the $\|\rightarrow \perp\|$ case where $\omega^{\prime}$ represents the final photon of $\perp$ polarization. Note that the magnitude of $\mathcal{M}_{\|\rightarrow \perp\|}$ diverges as pair threshold $w=2$ is approached.

The equivalent result for the splitting mode $\mathcal{M}_{\|\rightarrow \perp\|}$ requires little additional algebra given that it can be obtained from the analysis just above using the cyclic symmetry transformations of Eq. (19). Carefully keeping track of signs and all photon frequencies by relabelling at the beginning of the manipulations, the roles of the $\Delta_{4}$ and $\Delta_{3}$ terms are interchanged, and the obvious result emerges:

$$
\mathcal{M}_{\|\rightarrow \perp\|} \approx \frac{4 \omega^{\prime \prime}}{\omega \sqrt{4-\omega^{2}}} \arcsin \left(\frac{\omega}{2}\right)-\frac{4 \omega}{\omega^{\prime \prime} \sqrt{4-\left(\omega^{\prime \prime}\right)^{2}}} \arcsin \left(\frac{\omega^{\prime \prime}}{2}\right)+\omega^{\prime} \quad, \quad B \gg 1 .
$$

While not established before in the literature, the low energy limit of this, namely $\mathcal{M}_{\|\rightarrow \perp\|} \approx \omega \omega^{\prime} \omega^{\prime \prime} / 6$, yields the differential rate from previous expositions [4,6] of low energy approximations. The form of Eq. (36) is displayed in Figure 1, exhibiting the asymmetry expected under interchanges $\omega^{\prime} \leftrightarrow \omega^{\prime \prime}$. In this case, pair threshold structure in the amplitude appears again for the two photons of parallel polarization (i.e. at $\omega=2$ and $\omega^{\prime \prime}=2$ ), and is also absent for the produced $\perp$ photon, being of higher order in $B$. Consequently, the amplitude possesses a real divergence at $\omega=2$, a noteworthy occurrence that is illustrated by comparing the two panels of Figure 1 . Such divergences, which are not integrable over $\omega$ (and therefore patently different in nature from the resonances encountered in rates for $\gamma \rightarrow e^{ \pm}$), are characteristic of the photon splitting rate near resonances at and above the pair threshold of $\omega=2$, corresponding to the creation of virtual pairs in various excited states. In fact, near such resonances, photon splitting necessarily becomes first order in $\alpha_{\mathrm{f}}$ like pair creation as the intermediate states "go on-shell."

The rapid increase of the rate of $\|\rightarrow \perp\|$ relative to that of $\perp \rightarrow\|\| \|$ is exhibited in Figure 2, where the rates have been scaled by the low energy $(\omega \ll 1)$ limiting forms $\left(R(\omega) \propto \omega^{5}\right)$ discussed in the next subsection. This particular scaling is chosen to illustrate deviations from the $\omega \ll 1$ asymptotic forms, and therefore to demonstrate the need for relinquishing use of them when sampling photon energies near pair threshold, a parameter regime very relevant to certain astrophysical calculations (e.g. see [27,28]). The dominance of the $R_{\|\rightarrow \perp\|}$ over $R_{\perp \rightarrow\|\|}$ near $\omega=2$ apparent in these $B \gg 1$ results becomes substantive in parameter regimes where the weakly-dispersive vacuum (i.e. for

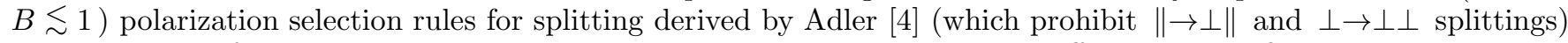
may not apply if non-linear contributions to vacuum polarization or plasma effects are significant. This underlines the saliency of a detailed determination of the dispersive properties of the magnetized vacuum or plasma medium appropriate to a particular astrophysical scenario.

The derivation of the $B \gg 1$ form for the amplitude for $\perp \rightarrow \perp \perp$ differs significantly from the results just expounded. First, contributions from $n^{\prime \prime}=1, n=n^{\prime}=0$ and $n^{\prime}=1, n=n^{\prime \prime}=0$ and $n=1, n^{\prime}=n^{\prime \prime}=0$ combinations are identically equal to zero by virtue of each of the associated $\Delta_{i}$ factors. This automatically implies that no inverse trigonometric functions that have arguments independent of $B$ appear in the amplitude, a property not possessed by the other splitting modes. The consequences of this are twofold. First, this cancellation implies that the scattering for 
$\perp \rightarrow \perp \perp$ is of a higher order in $B$ than for the other two splitting modes. Second, since any potential appearance of inverse trigonometric functions spawned by the forms in Eq. (33) involves arguments that depend on $B$ through the $\mathcal{N} \mathrm{s}$, these arguments are always small when $B \gg 1$, precipitating a redundancy with the low energy limit. Hence, it follows immediately that the scattering amplitude for $\perp \rightarrow \perp \perp$ in the regime of highly super-critical fields is identical to that of the $B \gg 1$ specialization of the low energy $(\omega \ll 1)$ limit. As the latter has been derived in various papers in the literature (e.g. see [4,6,11] and the subsequent section), here it is sufficient to merely state the result:

$$
\mathcal{M}_{\perp \rightarrow \perp \perp} \approx \frac{\omega \omega^{\prime} \omega^{\prime \prime}}{3 B} \quad, \quad B \gg 1 .
$$

This extremely simple form differs profoundly from those of the other two modes because of the absence of photons of $\|$ polarization in the interaction. Hence any signatures of the pair threshold of $1+\sqrt{1+2 B}$ of $\perp$ photons are absent in the domain of $\omega<2$, and a scaling-type form with obvious cyclic symmetry emerges.

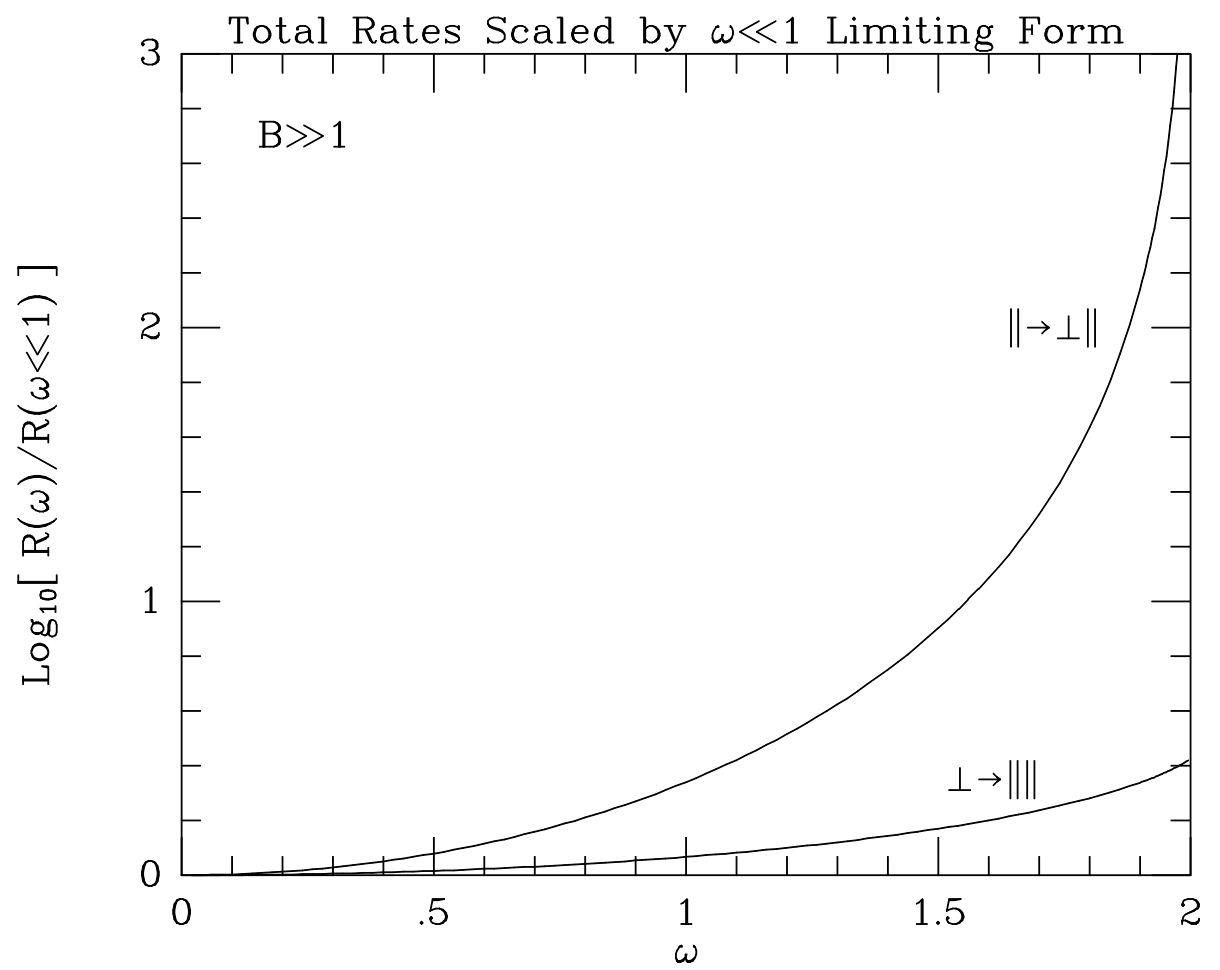

FIG. 2. The total rates in the $B \gg 1$ limit for the modes $\perp \rightarrow\|\|$ and $\|\rightarrow \perp\|$, computed according to Eq. (12) using the amplitude formulae in Eqs. (35) and (36), divided by the rates that would be computed when taking the low energy $(\omega \ll 1)$ limit of these amplitudes, i.e. $\mathcal{M}_{\perp \rightarrow\|\|} \approx \omega \omega^{\prime} \omega^{\prime \prime} / 6 \approx \mathcal{M}_{\|\rightarrow \perp\|}$. Deviations from such low energy approximations (i.e. $\left.R(\omega) \propto \omega^{5}\right)$, while significant for $\perp \rightarrow\|\|$, are dramatic for $\|\rightarrow \perp\|$ near pair creation threshold $w=2$.

\section{B. Approximations for $\omega \ll 1$}

The low energy limit $\omega \ll 1$ is of interest not only because it was the regime where compact analytic expressions for the splitting rates were first obtained [2 [4], but also because the analysis that follows derives simple and elegant representations of the scattering amplitudes in terms of well-known special functions that provide a convenient alternative option for numerical evaluations.

The amplitudes for each of the splitting modes should exhibit a cubic energy dependence $[$ 国 when $\omega \ll 1$. Hence, a necessary product of the Landau representation formalism is that terms linear in photon energies should contribute exactly zero. For the polarization modes $\perp \rightarrow\|\|$ and $\|\rightarrow \perp\|$, whose amplitudes are identical in the low energy limit [4.6, the demonstration of this is not dissimilar to the $B \gg 1$ analysis. The $\omega \ll 1$ restriction generates a single infinite series in $n$ due to the clustering of $n^{\prime}$ and $n^{\prime \prime}$ around $n$. The ensuing algebra in the simplification of this series is moderately lengthy, and requires re-indexing of the logarithmic terms to assume forms involving $\log _{e}[1+2 n B]$, and also some relabelling of the rational functions. Care must be taken in these rearrangements due to the infinite nature of the series, and the technique adopted is outlined just below. The terms linear in photon energy result in zero, as expected: for more details, the reader is referred to [11]. The next order contribution is cubic in energy, 
as desired, with terms coming from a mixture of (i) the linear terms of the $\Delta_{i}$ combined with the quadratic higher order terms of the $p_{z}$ integrals, and (ii) the cubic $\Delta_{i}$ terms in conjunction with the leading order (constant) terms from the $p_{z}$ integrals. The algebra is straightforward, but lengthy and tedious, generating an exact cancellation of all but terms proportional to $\omega \omega^{\prime} \omega^{\prime \prime}$. This approach leads to a reproduction of the $C_{j}$ listed in entirety in Appendix B of Weise, Baring \& Melrose [11] for both the $\perp \rightarrow\|\|$ and $\perp \rightarrow \perp \perp$ modes of splitting. Hence there is little point in replicating these expressions here; the reader is referred to [11] for details.

These results are expressed as single infinite series in the label $n$, which sometimes starts at $n=0$, and sometimes begins at higher integer values (up to 3 ). Hence, an aesthetic goal is to rearrange some of these series so that the summations in each contribution begin at $n=0$, and then add the terms in the series together. This is a nontrivial exercise, given the divergent nature of the series in many of the individual contributions. Hence, considerable care must be taken when performing the rearrangements, for which there is no unique prescription. One choice for relabelling the sums is adopted by Weise, Baring \& Melrose [1], though their end results expressed in their Appendix $\mathrm{C}$ do not facilitate analytic development in the most expedient manner, and were in fact erroneous (discussed briefly below). An alternative and preferable choice for rearrangement of the multitude of series over the label $n$ is adopted here, outlined as follows. Inspection of the various $C_{j}$ contributions in Appendix B of [1] reveals that they always consist of three types of terms: (i) logarithmic ones proportional to $\log _{e}[1+2(n+l) B]$, for $l=0, \pm 1, \pm 2, \pm 3$, (ii) rational functions of $1+2(n+l) B$ for $l=0, \pm 1, \pm 2$, and (iii) polynomials in $n$. A unique method for rearrangement is to truncate all series to finite ones with $n \leq k$, and then perform relabellings so that the first two types of terms consist only of $\log _{e}[1+2 n B]$ terms and rational functions of $1+2 n B$. This approach provides no particular focus on series that originate with labels $n>0$, but requires careful accounting of the remainder terms at the upper and lower ends of the sums, for which significant cancellation arises. The coefficients of the logarithmic functions, originally cubic in $n$, reduce to linear functions of $n$ in this development. The consequent simplification of the series terms is counterbalanced by the transferral of complexity to the constant remainder terms, which are purely functions of $k$ and $B$. Taking the limit of $k \rightarrow \infty$ achieves the desired (and convergent) result.

After considerable algebra collecting together all the constituent series in Appendix B of [11], and performing the rearrangement as just prescribed, one arrives at the following series representation of the scaled scattering amplitudes:

$$
\mathcal{M}_{e \rightarrow e^{\prime} e^{\prime \prime}}=\omega \omega^{\prime} \omega^{\prime \prime} \lim _{k \rightarrow \infty}\left\{\sum_{n=0}^{k} T_{e \rightarrow e^{\prime} e^{\prime \prime}}(n, B)+\mathcal{R}_{e \rightarrow e^{\prime} e^{\prime \prime}}(k, B)\right\}
$$

for $\omega \ll 1$, where

$$
\begin{aligned}
T_{\perp \rightarrow\|\| \|}(n, B) & =-\left(\frac{4 n}{B}+\frac{3}{2 B^{2}}\right) \log _{e}\left[n+\frac{1}{2 B}\right]+\left(\frac{2}{3}+\frac{1}{2 B^{2}}\right) \frac{1}{1+2 n B}-\frac{1}{3(1+2 n B)^{2}} \\
T_{\perp \rightarrow \perp \perp}(n, B) & =\frac{3}{2 B^{2}} \log _{e}\left[n+\frac{1}{2 B}\right]-\frac{3}{2 B^{2}} \frac{1}{1+2 n B}+\frac{1}{2 B^{2}} \frac{1}{(1+2 n B)^{2}}
\end{aligned}
$$

defines the series terms. The remainders are quite lengthy, and are listed in Appendix A.

Consider first the polarization mode $\perp \rightarrow \| \Perp$. While possibly only marginally simpler than Eq. (C1) of [11], the series and remainder in Eqs. (38), (39) and (A1) naturally enable the development of a special function representation of the scattering amplitude. The finite summation over terms like $(x+n) \log _{e}(x+n)$ in Eq. (39) can be expressed using result 44.1.2 of [36] in terms of an integral of the logarithm of the Gamma function. At this juncture, the analysis begins to image parts of that generated in expressing the polarization properties of a magnetized vacuum via effective Lagrangian or proper-time techniques [37 [39], as should be expected. Hence, it is appropriate to adopt definitions from such literature as much as possible. Following [37,38], here a definition for the generalized Gamma function $\Gamma_{1}(x)$ of

$$
\log _{e} \Gamma_{1}(x)=\int_{0}^{x} d t \log _{e} \Gamma(t)+\frac{1}{2} x(x-1)-\frac{x}{2} \log _{e} 2 \pi
$$

is adopted. Properties of this function, which include $\Gamma_{1}(1)=1$, are discussed at length in [40] and outlined in Appendix B.

Using Eq. (B1), one soon arrives at an expression for the scattering amplitude in terms of a handful of special (polygamma) functions, namely $\Gamma_{1}(x)$, and $\log _{e} \Gamma(x)$ and its derivatives. This representation consists of two parts, one independent of $k$, and one that involves a limit as $k \rightarrow \infty$ of the remainder in Eq. (A1), combined with several terms incorporating the special functions with arguments that depend on $k$. In evaluating this limit, most terms can be handled in a straightforward manner, and standard asymptotic series (e.g. see [33]) for $\log _{e} \Gamma(x)$ and $\psi(x)$ as $x \rightarrow \infty$ prove useful. However, the treatment of the term involving the function $\log _{e} \Gamma_{1}(1+k+1 / 2 B)$ that appears 
in the limit contribution is non-trivial. A series representation for this function for large arguments is required, and is presented in Eq. (B4). Assembling the various pieces, the limiting result as $k \rightarrow \infty$ is

$$
\begin{aligned}
\mathcal{M}_{\perp \rightarrow\|\|}=\omega \omega^{\prime} \omega^{\prime \prime}\left\{\frac{4}{B} \log _{e} \Gamma_{1}\left(\frac{1}{2 B}\right)-\frac{1}{2 B^{2}} \log _{e} \Gamma\left(\frac{1}{2 B}\right)-\left(\frac{1}{3 B}+\frac{1}{4 B^{3}}\right) \psi\left(\frac{1}{2 B}\right)\right. \\
\left.-\frac{1}{12 B^{2}} \psi^{\prime}\left(\frac{1}{2 B}\right)-\frac{1}{6}+\frac{1}{6 B}-\frac{4 L_{1}}{B}+\frac{1}{4 B^{2}}\left(\log _{e} 2 \pi-1-3 \log _{e} 2 B\right)\right\}
\end{aligned}
$$

for $\omega \ll 1$. This is the sought-after compact analytic form that is comparable in simplicity to the one-loop effective Lagrangians calculated in [37,38]. Using series and asymptotic expansions for all the special functions present, it is routine to establish that $\mathcal{M}_{\perp \rightarrow\|\|} \approx\left(26 B^{3} / 315\right) \omega \omega^{\prime} \omega^{\prime \prime}$ for $B \ll 1$, while for $B \gg 1$, one finds $\mathcal{M}_{\perp \rightarrow\|\|} \approx \omega \omega^{\prime} \omega^{\prime \prime} / 6$, a result obtainable from Eq. (35).

The developments are similar for the $\perp \rightarrow \perp \perp$ mode: this representation again consists of two parts, one independent of $k$, and one that involves a limit as $k \rightarrow \infty$ of the remainder in Eq. (A2), combined with several terms incorporating polygamma functions with arguments that depend on $k$. This limit can easily be evaluated using asymptotic series to yield (for $\omega \ll 1$ )

$$
\begin{aligned}
\mathcal{M}_{\perp \rightarrow \perp \perp}=\omega \omega^{\prime} \omega^{\prime \prime}\left\{-\frac{3}{2 B^{2}} \log _{e} \Gamma\left(\frac{1}{2 B}\right)+\frac{3}{4 B^{3}} \psi\left(\frac{1}{2 B}\right)\right. \\
\left.+\frac{1}{8 B^{4}} \psi^{\prime}\left(\frac{1}{2 B}\right)+\frac{1}{3 B}+\frac{1}{2 B^{2}}-\frac{1}{B^{3}}+\frac{3}{4 B^{2}}\left(\log _{e} 2 \pi+\log _{e} 2 B\right)\right\} .
\end{aligned}
$$

Using series and asymptotic expansions for all the special functions present, it is routine to establish that $\mathcal{M}_{\perp \rightarrow \perp \perp} \approx$ $\left(48 B^{3} / 315\right) \omega \omega^{\prime} \omega^{\prime \prime}$ for $B \ll 1$, while for $B \gg 1$, one finds $\mathcal{M}_{\perp \rightarrow \perp \perp} \approx \omega \omega^{\prime} \omega^{\prime \prime} /(3 B)$, the result stated in Eq. (37).

It must be remarked in passing that the expressions for $\perp \rightarrow \perp \perp$ in Eqs. (39), (A2) and (42) cannot be derived from the series in Eq. (C2) of 11], principally because that series expression is divergent, and therefore erroneous. Such an error was introduced by an inappropriate rearrangement of individually-divergent contributing series (leading to the addition of infinite contributions), a mistake that is avoided by the careful technique employed here in manipulating the results of Appendix B in [11]. Notwithstanding, the numerical results for the $\perp \rightarrow \perp \perp$ mode presented in [11] were effectively evaluated before any series rearrangement, and therefore remain valid.

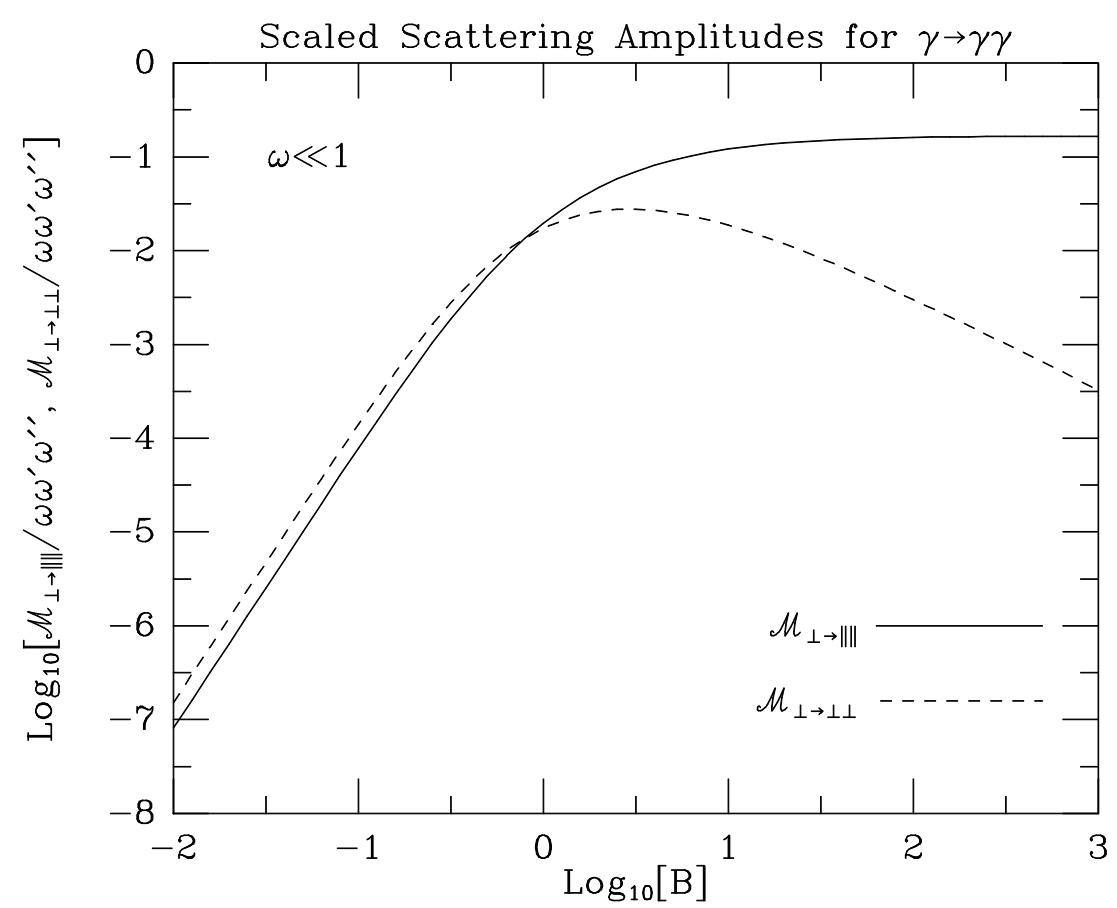

FIG. 3. The dependence of the scattering amplitudes, scaled by $\omega \omega^{\prime} \omega^{\prime \prime}$, on the magnetic field (in units of $B_{\mathrm{c}}=4.413 \times 10^{13}$ Gauss) for the splitting modes $\perp \rightarrow\|\|$ (see Eq. [41]) and $\perp \rightarrow \perp \perp$ (see Eq. [42]), for photon energies well below pair creation threshold. The amplitude for $\|\rightarrow \perp\|$ is identical to that for $\perp \rightarrow\|\|$. 
The compact analytic forms presented in Eqs. (41) and (42) represent the culmination of the $\omega \ll 1$ focus here. It is the first time such simple forms for the scattering amplitudes involving just special functions have been calculated in this limit, though somewhat more convoluted, yet essentially equivalent, expressions have been put forward in [41]. A distinct advantage of the expressions in Eqs. (41) and (42) is the ease with which they can be accurately computed numerically. Their dependence on $B$ is illustrated in Figure 3, replicating the numerics of [11] and earlier effective Lagrangian determinations [4], which are just as expedient since (see Eqs. [43] and [45] below) they involve just integrals of elementary functions.

The low frequency result for $\mathcal{M}_{\|\rightarrow \perp\|}$ is not presented explicitly since it reproduces that for $\mathcal{M}_{\perp \rightarrow\|\|}$ (e.g. see [1.6); this is due to the crossing symmetries involved. Note that while the cubic dependences of all the modes at low energies reflect the lack of an energy scale in this domain (i.e. such as pair threshold), the normalizations are dependent on the polarization mode, particularly at highly supercritical fields where the $\mathcal{M}_{\perp \rightarrow \perp \perp}$ amplitude is highly suppressed. This effectively represents how the rate normalization is sensitive to the (virtual) pair creation thresholds for the polarization states involved in a particular splitting mode.

To conclude this presentation focusing on the $\omega \ll 1$ specialization, an obvious objective is the re-derivation of Eqs. (41) and (42) starting with extant and well-known effective Lagrangian/proper-time (ELP) results, and thereby demonstrating analytically the equivalence of the S-matrix formulation in the Landau representation and Schwingertype formalisms in the low energy limit. Consider first the mode $\perp \rightarrow\|\|$, for which such a determination is somewhat involved. The starting point is the integral expression [4.6. 10] that corresponds to the scaled scattering amplitude that generates the same form for the rate as in Eq. (12):

$$
\mathcal{M}_{\perp \rightarrow\|\|}^{\mathrm{ELP}}=\frac{\omega \omega^{\prime} \omega^{\prime \prime}}{B} \int_{0}^{\infty} \frac{d s}{s} e^{-s / B}\left\{\left(-\frac{3}{4 s}+\frac{s}{6}\right) \frac{\cosh s}{\sinh s}+\frac{3+2 s^{2}}{12 \sinh ^{2} s}+\frac{s \cosh s}{2 \sinh ^{3} s}\right\},
$$

which has $B \ll 1$ and $B \gg 1$ limits matching those of Eq. (41). In the subsequent analysis, it is useful to manipulate integrations using the variable $\mu=1 / B$. The first step is to recognize that $1 / s^{3}$ times the factor in curly braces in Eq. (43) is a perfect derivative, namely $d g / d s$, where $g(s)=\left(1 / 4 s^{3}\right) d[s \operatorname{coth} s-1] / d s-\operatorname{coth} s /(6 s)$. Integration by parts is obviously the operative method, with the goal of retaining coth $s$ functions explicitly, combined with powers of $s$. After some algebra, one finds that

$$
\begin{gathered}
\frac{\mathcal{M}_{\perp \rightarrow\|\|}^{\mathrm{ELP}}}{\omega \omega^{\prime} \omega^{\prime \prime}}=\frac{1}{B} \int_{0}^{\infty} d s e^{-s / B}\left[\frac{1}{4 B^{2} s}-\frac{1}{4 B s^{2}}-\frac{1}{6 B}+\frac{1}{3 s}\right](s \operatorname{coth} s-1) \\
-\frac{1}{B} \int_{0}^{\infty} \frac{d s}{s^{3}} e^{-s / B}\left[s \operatorname{coth} s-1-\frac{s^{2}}{3}\right]
\end{gathered}
$$

results. The integral on the first line can be performed using identities 3.551.3 and 3.554.4 of [33], yielding the Gamma function and polygamma functions (or equivalently generalized Riemann Zeta functions) in addition to elementary functions. The only subtle part pertains to the second term of this integral, namely that contributed by the $-1 /\left(4 B s^{2}\right)$ factor. This can be differentiated with respect to $B$, evaluated to yield a $\psi$ function, and then the result integrated, noting the behaviour as $B \rightarrow 0$. The evaluation of the integral on the second line of Eq. (44) is much more involved. However, it has been performed before in the literature, and appears explicitly in calculations [37,38] of the one-loop effective Lagrangian describing refractive indices of the magnetized vacuum in QED. Hence the motivation for the particular partitioning of integrations chosen in Eq. (44). Details of the determination of this integral are found in Dittrich et al. [38], and the second line of Eq. (44) can be equated to $-8 \pi^{2} / B^{3}$ times the Lagrangian $\mathcal{L}^{(1)}(B)$ (see Eqs. (2.4) and (3.16) of [38]), thereby introducing the $\Gamma_{1}$ function. Collecting together the terms neatly generates an analytic form for $\mathcal{M}_{\perp \rightarrow\|\|}^{\mathrm{EDP}}$ that is identical to Eq. (41), so that the desired demonstration of equivalence of the Landau representation and effective Lagrangian forms is achieved.

The procedure for the $\perp \rightarrow \perp \perp$ mode is similar, though somewhat less involved. The equivalent scaled scattering amplitude obtained [4. [6] from effective Lagrangian/proper-time techniques is

$$
\mathcal{M}_{\perp \rightarrow \perp \perp}^{\mathrm{ELP}}=\frac{\omega \omega^{\prime} \omega^{\prime \prime}}{B} \int_{0}^{\infty} \frac{d s}{s} e^{-s / B}\left\{\frac{3}{4 s} \frac{\cosh s}{\sinh s}+\frac{3-4 s^{2}}{4 \sinh ^{2} s}-\frac{3 s^{2}}{2 \sinh ^{3} s}\right\} .
$$

Recognizing that the factor in curly braces can be written as $-(3 s / 4) d\left[\operatorname{coth} s / s-1 / s^{2}\right] / d s+\left(s^{2} / 4\right) d^{3}[\operatorname{coth} s-$ $1 / s] / d s^{3}$, integration by parts is again indicated, with identities 3.551.3 and 3.554.4 of [33] again proving useful. With manipulations similar to (but simpler than: the $\Gamma_{1}$ function is not involved here) those for the $\perp \rightarrow\|\|$, a modicum of algebra leads to the derivation of Eq. (42) from Eq. (45), as desired. This equivalence is a satisfying indication of the verity of the Landau representation analysis in this paper. 


\section{CONCLUSION}

This paper has provided a detailed development of the S-matrix formulation of the QED process of magnetic photon splitting in the Landau representation, focusing on the case of zero dispersion where photon propagation is collinear. The formalism in Section II rederives and extends the exposition of Mentzel, Berg \& Wunner [1]. The two principal general developments offered here are an analytic reduction via the summation over the spins of the intermediate pair states, discussed briefly in [11], and the analytic integration over the momenta parallel to the field incorporated in the electron propagators. This latter accomplishment is presented here for the first time. The cumulative product of these developments is a satisfyingly simple and elegant form in Eq. (15) for the scattering amplitude for each of the polarization modes permitted by CP invariance. These amplitudes possess products of generalized Laguerre polynomials that are common to QED processes in external magnetic fields, and elementary functions involving the photon energies and the various pair thresholds associated with the propagators. Moreover, the analytic forms presented consist of just triple summations over Landau level quantum numbers of the intermediate states, and are eminently suitable for accurate numerical computations both below and above pair creation threshold $\omega=2$. The applicability of these results to regimes above pair threshold is a benefit of the S-matrix expansion in the Landau representation that is not afforded by effective Lagrangian and proper-time calculations: while these (latter) Schwinger-type techniques elegantly formulate splitting rates below pair threshold, they eliminate the resonance structure early on in their mathematical developments, a severe limitation above $\omega=2$.

As an embellishment to these general results, specializations in two significant domains have been obtained. The first is for highly supercritical fields, $B \gg 1$, reproducing in particular the result of $\&$ for the $\perp \rightarrow\|\|$ mode, and deriving new results for the other two modes permitted by CP invariance in the limit of zero dispersion. The second group of asymptotic results are for energies $\omega \ll 1$ well below pair creation threshold, where new and compact expressions for the scattering amplitudes have been derived in Eqs. (41) and (42) in terms of the logarithm of the Gamma function, its integral and their derivatives. These two domains of specialization herein have facilitated the

first analytic demonstration of the equivalence of splitting rates obtained by the S-matrix formulation in the Landau representation and those derived using Schwinger-type effective Lagrangian/proper-time techniques.

\section{ACKNOWLEDGMENTS}

I thank my collaborators Jeanette Weise, Don Melrose and Alice Harding, and also Carlo Graziani and George Pavlov for many productive discussions, and also the referee Stephen Adler for suggestions that improved the presentation. I also thank the RCfTA at the University of Sydney for hospitality during part of the period when this work was performed.

\section{APPENDIX A:}

Here the remainders that appear in the series representation in Eq. (38) for the $\omega \ll 1$ specializations to the splitting amplitudes for polarization modes $\perp \rightarrow\|\|$ and $\perp \rightarrow \perp \perp$ are presented:

$$
\begin{aligned}
\mathcal{R}_{\perp \rightarrow\|\|}(k, B) & =\frac{k+1}{8 B}\left[2 k^{2}+k\left(4+\frac{1}{B}\right)+\frac{1}{B}\right] \log _{e}\left[k+2+\frac{1}{2 B}\right] \\
& +\frac{1}{4 B}\left[11 k^{3}+k^{2}\left(21+\frac{21}{2 B}\right)+k\left(10+\frac{14}{B}+\frac{5}{2 B^{2}}\right)+\frac{5}{2 B}+\frac{5}{4 B^{2}}\right] \log _{e}\left[k+1+\frac{1}{2 B}\right] \\
& -\frac{1}{4 B}\left[11 k^{3}+k^{2}\left(16+\frac{21}{2 B}\right)+k\left(5+\frac{9}{B}+\frac{5}{2 B^{2}}\right)+\frac{5}{4 B^{2}}\right] \log _{e}\left[k+\frac{1}{2 B}\right] \\
& -\frac{k}{4 B}\left[k^{2}+\frac{k}{2 B}-1\right] \log _{e}\left[k-1+\frac{1}{2 B}\right] \\
& -\left(\frac{B}{3}(k+1)+\frac{1}{6}+\frac{k+1}{2 B}\right) \frac{1}{1+2(k+1) B}+\frac{k}{2 B[1+2 k B]} \\
& -\frac{9 k^{2}}{2 B}-\frac{5 k}{B}-\frac{7 k}{4 B^{2}}-\frac{5}{8 B}-\frac{9}{8 B^{2}}-\frac{3}{4 B^{2}} \log _{e} 2 B
\end{aligned}
$$

and 


$$
\begin{aligned}
\mathcal{R}_{\perp \rightarrow \perp \perp}(k, B)= & -\frac{(k+1)(k+2)(k+3)}{12 B} \log _{e}\left[k+3+\frac{1}{2 B}\right] \\
& -\frac{k+1}{16 B}\left[\frac{13}{3} k^{2}+k\left(\frac{32}{3}+\frac{7}{2 B}\right)+4+\frac{7}{2 B}\right] \log _{e}\left[k+2+\frac{1}{2 B}\right] \\
& +\frac{1}{16 B}\left[\frac{71}{3} k^{3}+k^{2}\left(38+\frac{9}{2 B}\right)+k\left(\frac{79}{3}-\frac{4}{B}\right)+12-\frac{2}{B}\right] \log _{e}\left[k+1+\frac{1}{2 B}\right] \\
& -\frac{1}{16 B}\left[\frac{71}{3} k^{3}+k^{2}\left(13+\frac{9}{2 B}\right)-k\left(\frac{20}{3}-\frac{13}{B}\right)+\frac{13}{2 B}\right] \log _{e}\left[k+\frac{1}{2 B}\right] \\
+ & \frac{k}{16 B}\left[\frac{13}{3} k^{2}+k\left(2+\frac{7}{2 B}\right)-\frac{19}{3}\right] \log _{e}\left[k-1+\frac{1}{2 B}\right] \\
& +\frac{k(k-1)(k-2)}{12 B} \log _{e}\left[k-2+\frac{1}{2 B}\right]+\frac{(k+1)(k+2)^{2}}{2[1+2(k+2) B]} \\
& -\frac{(k+1)^{2}(5 k+2)}{2[1+2(k+1) B]}-\frac{k^{2}(5 k-1)}{2[1+2 k B]}+\frac{k(k-1)^{2}}{2[1+2(k-1) B]} \\
& +\frac{7 k^{2}}{4 B}+\frac{7 k}{4 B}+\frac{9}{8 B}+\frac{k+1}{B^{2}}+\frac{3}{4 B^{2}} \log _{e} 2 B .
\end{aligned}
$$

\section{APPENDIX B:}

In this Appendix, various useful properties of the $\Gamma_{1}(x)$ function, the integral of the logarithm of the Gamma function, that are needed in the $\omega \ll 1$ specializations are stated. Given the definition of $\Gamma_{1}$ in Eq. (40), it is elementary to establish, using 44.1 .2 of [36], that

$$
\sum_{n=0}^{k}(x+n) \log _{e}(x+n)=\log _{e} \Gamma_{1}(1+x+k)-\log _{e} \Gamma_{1}(x) .
$$

Taking successive derivatives with respect to $x$, one quickly arrives at well-known finite series representations of $\Gamma(x)$ and its logarithmic derivative $\psi(x)$; see [33,42 for discussions of these functions and their series representations.

An asymptotic series representation for the $\Gamma_{1}$ function for large arguments is useful, and can be derived with the aid of the following series representation (see result 8.343.2 of [33]) for the logarithm of the Gamma function:

$$
\log _{e} \Gamma(x)=\left(x-\frac{1}{2}\right) \log _{e} x-x+\frac{1}{2} \log _{e} 2 \pi+\frac{1}{2} \sum_{m=1}^{\infty} \frac{m}{(m+1)(m+2)}\left[\zeta(m+1, x)-\frac{1}{x^{m+1}}\right]
$$

from which Stirling's asymptotic expansion can be derived. Here, $\zeta(m, t)$ is the generalized Riemann Zeta function, defined in 9.511 and 9.521.1 of [33]. The integration of this series is effected using the identity $\zeta^{\prime}(m, t)=-\zeta(m+1, t)$, and is mostly uneventful. However, the treatment of the $m=1$ term in the summation is somewhat more subtle, due to the singular nature of $\zeta(0, t)$, and requires taking the limit $m \rightarrow 1^{+}$, assuming $m$ to be a continuous variable. Then result 8.362 .1 of [33] comes in handy, and the series identity

$$
\begin{aligned}
\log _{e} \Gamma_{1}(x)=\frac{x-1}{4}\left(2 x \log _{e} x-x-1\right)+\frac{1}{12}\left[\psi(x)+\frac{1}{x}+\gamma_{\mathrm{E}}-1\right] \\
+\frac{1}{2} \sum_{m=2}^{\infty} \frac{1}{(m+1)(m+2)}\left[\zeta(m, 1)-\zeta(m, x)-1+\frac{1}{x^{m}}\right]
\end{aligned}
$$

follows, where $\gamma_{\mathrm{E}}=-\psi(1) \approx 0.5772$ is Euler's constant. This series, which adequately substitutes for an asymptotic representation, can be used very effectively for numerical evaluations for all $x \geq 1$. For the range $0 \leq x<1$, this series also effects accurate evaluation of $\log _{e} \Gamma_{1}(x)$ via use of the recurrence relation $\log _{e} \Gamma_{1}(x)=\log _{e} \Gamma_{1}(1+x)-x \log _{e} x$, an identity derivable from Eq. (40) with the aid of 6.441 .3 in [33]. For large $x$, it then follows that

$$
\log _{e} \Gamma_{1}(x) \sim\left(\frac{x(x-1)}{2}+\frac{1}{12}\right) \log _{e} x-\frac{x^{2}}{4}+L_{1}+O\left(\frac{1}{x}\right)
$$


where

$$
\begin{aligned}
L_{1} & =\frac{\gamma_{\mathrm{E}}}{12}+\frac{1}{6}+\frac{1}{2} \sum_{m=2}^{\infty} \frac{\zeta(m, 1)-1}{(m+1)(m+2)} \\
& \equiv \lim _{k \rightarrow \infty}\left\{\sum_{n=1}^{k} n \log _{e} n-\left(\frac{k(k+1)}{2}+\frac{1}{12}\right) \log _{e} k+\frac{k^{2}}{4}\right\}
\end{aligned}
$$

with numerical value $L_{1} \approx 0.24875$. This is just the constant appearing in the magnetized vacuum polarization analyses of [37, 38], where the Raabe integral form for it can be found. The second definition of $L_{1}$ in Eq. (B5) can be obtained by setting $x=0$ in Eq. (B1), and is a result noted by [41].

[1] M. Mentzel, D. Berg \& G. Wunner, Phys. Rev. D50, 1125 (1994).

[2] S. L. Adler, J. N. Bahcall, C. G. Callan \& M. N. Rosenbluth, Phys. Rev. Lett. 25, 1061 (1970).

[3] Z. Bialynicka-Birula \& I. Bialynicki-Birula, Phys. Rev. D2, 2341 (1970).

[4] S. L. Adler, Ann. Phys. NY 67, 599 (1971).

[5] V. O. Papanyan \& V. I. Ritus, Sov. Phys. JETP 34, 1195 (1972).

[6] R. J. Stoneham, J. Phys. A12, 2187 (1979).

[7] V. N. Baier, A. I. Milstein \& R. Zh. Shaisultanov, Sov. Phys. JETP 63, 665 (1986).

[8] V. N. Baier, A. I. Milstein \& R. Zh. Shaisultanov, Phys. Rev. Lett. 77, 1691 (1996).

[9] S. L. Adler \& C. Schubert, Phys. Rev. Lett. 77, 1695 (1996).

[10] M. G. Baring \& A. K. Harding, Astrophys. J. 482, 372 (1997).

[11] J. I. Weise, M. G. Baring \& D. B. Melrose, Phys Rev. D. 57, 5526 (1998).

[12] C. Wilke \& G. Wunner, Phys. Rev. D. 55, 997 (1997).

[13] D. B. Melrose \& A. J. Parle, Aust. J. Phys. 36, 775 (1983).

[14] D. B. Melrose \& A. J. Parle, Aust. J. Phys. 36, 799 (1983).

[15] D. B. Melrose \& A. J. Parle, Aust. J. Phys. 36, 755 (1983).

[16] W.-Y. Tsai \& T. Erber, Phys. Rev. D. 10, 492 (1974).

[17] J. K. Daugherty \& A. K. Harding, Astrophys. J. 273, 761 (1983).

[18] Mitrofanov, I. G., et al., Sov. Astron. 30, 659 (1986).

[19] Baring, M. G., MNRAS 235, 79 (1988).

[20] M. G. Baring, Astrophys. J. Lett. 440, L69 (1995).

[21] M. G. Baring \& A. K. Harding, Astr. Space Sci. 231, 77 (1995).

[22] A. K. Harding \& M. G. Baring, Proc. Huntsville GRB Workshop (AIP 384, New York), p. 941 (1996).

[23] H.-K. Chang, K. Chen, E. E. Fenimore, \& C. Ho, Proc. Huntsville Workshop on Gamma-Ray Bursts, (AIP 384, New York), p. 921 (1996).

[24] Kouveliotou, C., et al., Nature 393, 235 (1999).

[25] Hurley, K., et al., Nature 397, 41 (1999).

[26] Kouveliotou, C., et al., Astrophys. J. Lett. 510, L116 (1999).

[27] M. G. Baring \& A. K. Harding, Astrophys. J. Lett. 507, L55 (1998).

[28] A. K. Harding, M. G. Baring \& P. L. Gonthier, Astrophys. J. 476, 246 (1997).

[29] A. A. Sokolov \& I. M. Ternov, Synchrotron Radiation (Pergamon, Oxford, 1968).

[30] H. Herold, H. Ruder \& G. Wunner, Astr. Astrophys. 115, 90 (1982).

[31] Shabad, A. E., Ann. Phys. 90, 166 (1975).

[32] Bulik, T. \& Miller, M. C., MNRAS 288, 596 (1997).

[33] I. S. Gradshteyn \& I. M. Ryzhik, Table of Integrals, Series, and Products (Academic, New York, 1980).

[34] D. B. Melrose \& R. J. Stoneham, J. Phys. A. 10, 1211 (1977).

[35] G. G. Pavlov, Yu. A. Shibanov \& D. G. Yakovlev, Astr. Space Sci. 73, 33 (1980).

[36] E. R. Hansen, A Table of Series and Products (Prentice-Hall, Englewood Cliffs, NJ).

[37] W.-Y. Tsai \& T. Erber, Phys. Rev. D. 12, 1132 (1975).

[38] W. Dittrich, W.-Y. Tsai, \& K.-H. Zimmermann, Phys. Rev. D. 19, 2929 (1979).

[39] B. V. Ivanov, Physics Lett. B 282, 228 (1992).

[40] L. Bendersky, Acta Math. 61, 263 (1933).

[41] J. S. Heyl \& J. Hernquist, Phys Rev. D. 55, 2449 (1997).

[42] A. Erdélyi, Higher Transcendental Functions, Volumes I-III, (Krieger Publishing, Malabar, Florida) 